\title{
Simple and collective twisted symmetries
}

G. Gaeta

To cite this article: G. Gaeta (2014) Simple and collective twisted symmetries, Journal of Nonlinear Mathematical Physics 21:4, 593-627, DOI:

https://doi.org/10.1080/14029251.2014.975530

To link to this article: https://doi.org/10.1080/14029251.2014.975530

Published online: 04 January 2021 


\title{
Simple and collective twisted symmetries
}

\author{
G. Gaeta* \\ Dipartimento di Matematica, Università degli Studi di Milano, \\ via C. Saldini 50, 20133 Milano (Italy) \\ giuseppe.gaeta@unimi.it
}

Received 8 May 2014

Accepted 23 August 2014

\begin{abstract}
After the introduction of $\lambda$-symmetries by Muriel and Romero, several other types of so called "twisted symmetries" have been considered in the literature (their name refers to the fact they are defined through a deformation of the familiar prolongation operation); they are as useful as standard symmetries for what concerns symmetry reduction of ODEs or determination of special (invariant) solutions for PDEs and have thus attracted attention. The geometrical relation of twisted symmetries to standard ones has already been noted: for some type of twisted symmetries (in particular, $\lambda$ and $\mu$-symmetries), this amounts to a certain kind of gauge transformation.

In a previous review paper [23] we have surveyed the first part of the developments of this theory; in the present paper we review recent developments. In particular, we provide a unifying geometrical description of the different types of twisted symmetries; this is based on the classical Frobenius reduction applied to distribution generated by Lie-point (local) symmetries.
\end{abstract}

Keywords: Symmetry; Differential Equations.

2000 Mathematics Subject Classification: 34A26, 35A30, 58J70

\section{Introduction}

Symmetry analysis of differential equations is a classical topic at least since the times of Sophus Lie, who indeed created what is nowadays known as the theory of Lie groups as a tool to analyze differential equations. In particular, symmetry properties are used both to obtain (symmetry) reduction of ODEs and special (symmetric) solutions to PDEs. The general standard scheme of Lie group analysis of differential equations $[1,15,32,55,56,63]$ goes with considering the phase manifold $M=B \times U$, where $B$ is the manifold of independent variables $x^{i}$ and $U$ that of dependent variables $u^{a}$, and Lie-point transformations acting in this. Once we know how the $x$ and the $u$ are transformed, we also know how the derivatives (of the $u^{a}$ with respect to the $x^{i}$, of any order) are transformed, so that the transformation considered in $M$ is readily prolonged to a transformation in the Jet bundle $J^{n} M$ (see Section 1 below for detail). Having defined a transformation in the space of $x, u$, and derivatives of any order - in particular, up to the order of the differential equation or system under study - we can ascertain if the transformation leaves the equation invariant, in which case it qualifies as a symmetry of the latter, and can be used for symmetry reduction or for determining special invariant solutions. This scheme was generalized in several directions as for the kind of transformations to be considered in $M$ as a starting point for the whole procedure (e.g., Lie himself

*Research partially supported by MIUR-PRIN program under project 2010-JJ4KPA 
considered contact transformations, in which the change of $x, u$ depends on the first order derivatives as well; more generally one can consider generalized maps depending on derivatives of order $m$ ); but a firm point of the analysis is that once the map acting in $M$ is given, the action on derivatives follows from that on basic variables. It came thus as a surprise when, about ten years ago, Muriel and Romero [40,41] came out with a different proposal: that is, consider standard Lie-point transformation in $M$, but deform the prolongation operation. The deformation they considered depends on a smooth function $\lambda: J^{1} M \rightarrow \mathbf{R}$, so that they christened these as $\lambda$-prolongations and, when the prolonged vector field in $J^{n} M$ leaves the equation invariant, $\lambda$-symmetries; standard ones are recovered for $\lambda=0$, so that we have an extension of standard Lie-point symmetries. What was more relevant, they showed that such $\lambda$-symmetries were as useful as standard ones for the symmetry reduction of ODEs (and systems thereof). As $\lambda$-symmetries are more general than standard ones, this opens the way at the possibility of applying symmetry techniques - in particular, symmetry reduction, and maybe a full solution - to equations with a lack of standard symmetries. This was indeed the motivation behind the work of Muriel and Romero, and in their first works they were immediately able to obtain concrete results in this direction. The approach followed for the creation of $\lambda$-symmetry was inherently analytic; but the theory of symmetries of differential equations is deeply rooted in Geometry (of Jet bundles), and a geometric understanding was needed. A first step in this direction was obtained by Pucci and Saccomandi [60], who noted that $\lambda$-prolonged vector field have a peculiar characterization, being collinear to the standard prolongation of some (in general, different) vector field in $M$. This opened the way to a geometric understanding of the matter; it turns out that the ODEs case is a degenerate one in this respect, and hides several features, so that a full understanding is better reached in the more general case of PDEs. In this framework the smooth function $\lambda$ is replaced by a semi-basic one form $\mu=\Lambda_{i} \mathrm{~d} x^{i}$ in $J^{1} M$, the $\Lambda_{i}$ being smooth matrix functions (their dimension being the same as the dimension of $U$ ), and one speaks accordingly of $\mu$-prolongations and $\mu$-symmetries $[16,25]$. The $\Lambda_{i}$ have to obey some compatibility condition (see Sect.1.5 below), but in the ODE case there is only one $\Lambda$, hence no compatibility. The special case of $\mu$-symmetries for ODEs has been studied in detail by Cicogna [10-12]; to emphasize its intermediate character one speaks of $\Lambda$-symmetries, and sometimes of $\rho$-symmetries when dealing with the class of $\Lambda$-symmetries which effectively leads to a reduction of ODEs (in this case the $\rho$ stands for "reducing"). The collective name for these deformed prolongations and symmetries is that of twisted prolongations and symmetries. These developments, and their geometrical aspects, were reviewed in detail in a previous work by the present author [23], describing the situation about five years ago and in particular the connection with gauge transformations. In the present paper, we want to review further and more recent progress. This focuses in particular on the possibility of considering deformed prolongations not of a single vector field but of a set (in involution) of vector fields, each of them entering in the prolongation of the others. This induces naturally to focus on the (Frobenius) distributions generated by the set of vector fields, and this shift of focus allows to finally reach a sound understanding of the geometrical aspects of twisted prolongations, explaining the reason for the appearance of gauge transformations.

Acknowledgements. I thank the referees for useful remarks and suggestions. My research is partially supported by MIUR-PRIN program under project 2010-JJ4KPA. 


\section{Background material}

We start by recalling the usual symmetry reduction procedure (under standard symmetries) for ODEs or systems thereof; this will allow to fix notation and be useful for later reference. We refer to [55] or also e.g. [1, 15, 22, 32, 54, 56, 63] for details. On the other hand, we assume the reader is familiar with the basic notions in the theory of symmetry of differential equations (and its use for solving these); see e.g. again $[1,15,32,55,56,63]$.

\subsection{General framework}

We denote the system (which could reduce to a single equation) of ODEs under study as $\Delta$, and assume for simplicity that it is in standard form, i.e. is solved w.r.t. higher order derivatives. We will write the independent variable as $x$ and the dependent ones as $u^{a}(a=1, \ldots, p)$; derivatives of these will be denoted as $u_{x}^{a}, u_{x x}^{a}$, and so on. We will also use the notation

$$
u_{(n)}^{a}:=\frac{d^{n} u^{a}}{d x^{n}}
$$

We thus have $\Delta$ written in the form

$$
u_{(n)}^{a}=F^{a}\left(x, u, \ldots, u_{(n-1)}\right) .
$$

The extended phase manifold will be $M=B \times U$, where $B=\mathbf{R}$ (with $x \in B$ ) and $u=\left(u^{1}, \ldots, u^{p}\right)$ takes values in $U \subseteq \mathbf{R}^{p}$. We consider Lie-point vector fields in $M$; these are written in coordinates as

$$
X=\xi(x, u) \frac{\partial}{\partial x}+\varphi^{a}(x, u) \frac{\partial}{\partial u^{a}} \equiv \xi \partial_{x}+\varphi^{a} \partial_{a} .
$$

Here and in the following, we use the Einstein sum convention.

As well known, (1.1) identifies a submanifold $S_{\Delta}$ in the $n$-th order Jet space over $M, S \subset J^{(n)} M$; this is called the solution manifold for $\Delta$. It is also well known that $X$ induces a vector field $X^{(n)}$ in $J^{(n)} M$ (this is also known as the prolongation of $X$ ); then $X$ is a Lie-point symmetry of $\Delta$ if and only if

$$
X^{(n)}: S_{\Delta} \rightarrow \mathrm{T} S_{\Delta} .
$$

The same relation can be expressed algebraically, considering $X$ and $X^{(n)}$ as differential operators, as

$$
\left[X^{(n)}(\Delta)\right]_{S_{\Delta}}=0
$$

It is convenient, for later reference, to recall how $X^{(n)}$ is built from $X$. With the notation already introduced, we write

$$
X^{(n)}=\xi \frac{\partial}{\partial x}+\psi_{(n)}^{a} \frac{\partial}{\partial u_{(n)}^{a}}
$$

then the prolongation formula is given in recursive terms as

$$
\psi_{(n+1)}^{a}=D_{x}\left(\psi_{(n)}^{a}\right)-u_{(n+1)}^{a} D_{x} \xi,
$$


G. Gaeta

where $\psi_{(0)}^{a}=\varphi^{a}$; here and below $D_{x}$ is the total derivative operator (with respect to $x$ ),

$$
D_{x}=\frac{\partial}{\partial x}+u_{x}^{a} \frac{\partial}{\partial u^{a}}+u_{x x}^{a} \frac{\partial}{\partial u_{x}^{a}}+\ldots=\frac{\partial}{\partial x}+u_{(k+1)}^{a} \frac{\partial}{\partial u_{(k)}^{a}} .
$$

We also recall, again for later reference, that to the vector field $X$ is associated its evolutionary representative $X_{v}$; with $X$ as in (1.2), this is defined by

$$
X_{v}=\left(\varphi^{a}-u_{x}^{a} \xi\right) \frac{\partial}{\partial u^{a}}:=Q^{a} \frac{\partial}{\partial u^{a}} .
$$

As well known, $X^{(n)}: S_{\Delta} \rightarrow \mathrm{T} S_{\Delta}$ if and only if $X_{v}^{(n)}: S_{\Delta} \rightarrow \mathrm{T} S_{\Delta}$ a . In other words $X$ is a symmetry of $\Delta$ if and only if $X_{v}$ is a symmetry for the same system $\Delta$ (see Proposition 5.5 in [55]).

It is easy to check by explicit computations (e.g. in recursive form) that

$$
Q_{(k)}^{a}=\psi_{(k)}^{a}-u_{(k+1)}^{a} \xi .
$$

It follows from this that the prolongation of a vector field $X$ of the form (1.2) and of its evolutionary representative $X_{v}=Q^{a} \partial_{a}$ are related through

$$
X_{v}^{(k)}=X^{(k)}-\xi \partial_{x}-\left[u_{(k+1)}^{a} \xi\right] \partial_{a}^{k} .
$$

Finally, we say that $X$ is a strong symmetry for $\Delta$ if $X^{(n)}(\Delta)=0$; i.e., at difference with (1.3) and (1.4), we do not consider restriction to $S_{\Delta}$.

Remark 1. It may be proved that if $X$ is a (standard) Lie-point symmetry for $\Delta$, then there is an equivalent equation $\widetilde{\Delta}$ which admits $X$ as a strong symmetry [6]. Equivalence here is meant as admitting the same set of solutions; in other words, we have

$$
\Delta=e^{\mathscr{F}} \widetilde{\Delta}
$$

with $\mathscr{F}=\mathscr{F}\left(x ; u, \ldots, u_{(n)}\right)$. Such a $\widetilde{\Delta}$ is necessarily written in terms of the differential invariants for $X$ [55], see below.

Let us now consider solutions to (a system of) differential equations; a function $u=f(x), u: B \rightarrow$ $U$, is identified with its graph, i.e. with a section $\gamma_{f}$ of the bundle $\left(M, \pi_{0}, B\right)$ with fiber $\pi_{0}^{-1}(x) \simeq U$. This is just

$$
\gamma_{f}=\{(x, u) \in M: u=f(x)\} .
$$

The section is naturally lifted (or prolonged) to a section $\gamma_{f}^{(n)}$ of $\left(J^{(n)} M, \pi_{n}, B\right)$, i.e. of $J^{(n)} M$ seen as a bundle over $B$; here $\pi_{n}^{-1}(x)=\left(u, u_{(1)}, \ldots, u_{(n)}\right)$.

The function $u=f(x)$ is a solution to the equation $\Delta$ of order $n$ if and only if $\gamma_{f}^{(n)} \subset S_{\Delta}$. Thus a symmetry of $\Delta$ maps solutions into (generally, different) solutions. (This can also be taken as the definition of symmetries.)

We say that $u=f(x)$ is an invariant solution (under $X$ ) to $\Delta$ if it is a solution and $X: \gamma_{f} \rightarrow \mathrm{T} \gamma_{f}$. This condition is completely equivalent to requiring that $X^{(n)}: \gamma_{f}^{(n)} \rightarrow \mathrm{T} \gamma_{f}^{(n)}[1,15,32,55,56,63]$.

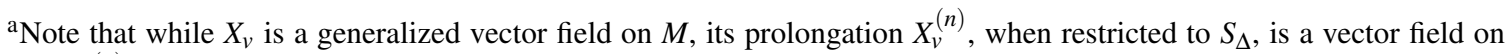
$S_{\Delta} \subset J^{(n)} M$. 
Similarly, if we consider a Lie algebra $\mathscr{G}$ of vector fields in $M$, we say that $u=f(x)$ is a $\mathscr{G}$-invariant solution to $\Delta$ if it is a solution and $X: \gamma_{f} \rightarrow \mathrm{T} \gamma_{f}$ for all $X \in \mathscr{G}$.

Remark 2. We stress that when requiring e.g. $X: \gamma_{f} \rightarrow \mathrm{T} \gamma_{f}$, we are considering $\gamma_{f}$ as a subset of $M$; if we consider $\gamma_{f}$ as a point in the space $\Sigma\left(M, \pi_{0}, B\right)$ of smooth sections of $\left(M, \pi_{0}, B\right)$, and denote by $\Phi_{X}$ the flow induced by $X$ in this space, we would rephrase this requirement as $\Phi_{X}: \gamma_{f} \rightarrow \gamma_{f}$.

\subsection{Symmetry reduction of ODEs}

Let us now recall the standard symmetry reduction procedure for ODEs; this is based on adapted coordinates. For the sake of simplicity, we will first discuss the case where $\Delta$ is a single ODE of order $n, U \simeq \mathbf{R}$ and $M \simeq \mathbf{R}^{2}$. We assume that $X$ is a Lie-point symmetry for $\Delta$, thus

$$
\left[X^{(n)}(\Delta)\right]_{S_{\Delta}}=0
$$

Now the key idea is to pass to new coordinates $(y, v)$ in $M$ (where $y$ should be thought as the independent variable) such that in the new coordinates $X$ is written as

$$
X=\frac{\partial}{\partial v} .
$$

(Alternatively, it may suffice to get coordinates such that $X=\psi(y, v)(\partial / \partial v)$, with $\psi$ nonzero; this requires a more detailed discussion.)

The point is that as symmetry is a tangency condition, see (1.3), and such a condition is independent of the coordinate description, we are guaranteed that expressing $\Delta$ in the new coordinates we still have (1.3); to stress we are expressing $\Delta$ in the new coordinates ${ }^{\mathrm{b}}$, we will also write it as $\widehat{\Delta}$.

On the other hand, (1.10) immediately entails that

$$
X^{(n)}=\frac{\partial}{\partial v}=X
$$

thus $\widehat{\Delta}$ admits $X$ as a symmetry if and only if it can be written with no explicit dependence on $v$. In other words, the (new) coordinate expression of our equation will be

$$
\frac{\partial^{n} v}{\partial y^{n}}=G\left(y ; v, v_{(1)}, \ldots, v_{(n-1)}\right)
$$

with $G$ actually independent of $v$,

$$
\partial G / \partial v=0
$$

We can thus rewrite $\widehat{\Delta}$ as

$$
\frac{\partial^{n} v}{\partial y^{n}}=G\left(y ; v_{(1)}, \ldots, v_{(n-1)}\right)
$$

It suffices to pass to the new variable

$$
w:=v_{(1)}=(\partial v / \partial y)
$$

\footnotetext{
${ }^{\mathrm{b}}$ We trust there is no possible confusion between the equation as a geometrical object (a submanifold in Jet space) and its coordinate expression.
} 
to get an equation of order $(n-1)$, i.e. to rewrite $\widehat{\Delta}$ as

$$
\frac{\partial^{n-1} w}{\partial y^{n-1}}=G\left(y ; w, w_{(1)}, \ldots, w_{(n-2)}\right) .
$$

Note that if we have a solution to (1.15), this provides solutions to the original equation $\Delta$ : in fact, (1.14) yields $v$ just by a quadrature (which introduces an integration constant), and this gives a solution to $\widehat{\Delta}$. Then we just have to invert the change of variables $(x, u) \rightarrow(y, v)$ to get a solution to $\Delta$.

In the case of a system, a Lie-point symmetry allows in this way to reduce by one the order of one of the equations. In the case where we have several Lie-point symmetries with a favorable Lie-algebraic structure (e.g. they should form a solvable algebra) we can correspondingly reduce several equations in the system, or reduce the order of a single equation by several degrees. We refer to $[1,15,32,55,56,63]$ for further detail.

Remark 3. Note that the reduction approach can be improved by considering so called solvable structures; the reader is referred to $[2,3,30,62]$ for detail on these (see also [8,9,39] for recent results bearing connection to our topic).

\subsection{Differential invariants and IBDP}

The discussion of the previous subsection is not entirely satisfactory, in that it is based on coordinate representations. In order to make it coordinate-free, we should remark that a differential equation admitting a given vector field $X$ as a Lie-point symmetry should be written in terms of the differential invariants for $X$, possibly plus a nowhere vanishing non-invariant term (see subsection 1.2, in particular Remark 1, above).

Thus - up to the nowhere vanishing and thus irrelevant (in the search for solutions) term, which we can write as $e^{\mathscr{F}}-$ the equation $\Delta$ is written as

$$
\Delta=\Phi\left[\eta, \zeta_{(1)}, \ldots, \zeta_{(n)}\right]
$$

where $\zeta_{(k)}: J^{(k)} M \rightarrow \mathbf{R}$ are the differential invariants of order $k$, and $\eta: M \rightarrow \mathbf{R}$ invariants of order zero (geometrical invariants).

It is essential to note that once we have differential invariants of order one, those of higher order can be obtained simply by derivation. In fact, we have the so called "invariant by differentiation property" (IBDP); this is well known and we give it without proof (for a proof see e.g. [55]; see also our proof of Lemma 5 below). ${ }^{\mathrm{c}}$

Lemma 1 (IBDP). Let $\eta$ be a geometrical invariant for $X$, and $\zeta_{(k)}$ a differential invariant of order $k$ for $X$. Then

$$
\zeta_{(k+1)}:=\frac{D_{x} \zeta_{(k)}}{D_{x} \eta}
$$

is a differential invariant of order $k+1$ for $X$.

$\overline{{ }^{c}}$ The relevance of IBDP for twisted symmetries was recognized since the beginning; e.g. Muriel and Romero emphasized it for $\lambda$-symmetries in [42]. 


\subsection{Multiple symmetries}

We have so far considered a single vector field; we could of course have several Lie-point symmetries $X_{i}$, and in view of (1.3) it is clear that the prolonged vector fields $X_{i}^{(n)}=Y_{i}$ will form a Lie algebra. That is, if $\mathscr{Y}_{\Delta}$ is the set of vector fields in $J^{n} M$ which satisfy (1.3), then $Y_{i} \in \mathscr{Y}_{\Delta}, Y_{j} \in \mathscr{Y}_{\Delta}$ implies that $\left[Y_{i}, Y_{j}\right] \in \mathscr{Y}_{\Delta}$ as well.

The possibility of using, at least in principles ${ }^{\mathrm{d}}$, all the symmetry vector fields to obtain a reduction of the $\Delta$ under study, and actually the very possibility of speaking of a symmetry algebra for $\Delta$, rely upon the following well known fact (this is Theorem 2.39 in [55]; see the proof in there):

Lemma 2. Let $X_{i}$ be Lie-point vector fields on $M$, and $X_{i}^{(n)}$ their prolongation on $J^{n} M$. The prolongation of a commutator is the commutator of the prolongations, so the $X_{i}^{(n)}$ satisfy the same Lie-algebraic relations as the $X_{i}$ :

$$
\left[X_{i}^{(n)}, X_{j}^{(n)}\right]=\left(\left[X_{i}, X_{j}\right]\right)^{(n)}
$$

\subsection{Invariant solutions for PDEs}

The situation is quite different when we analyze PDEs. Albeit the results mentioned above do hold also in this case, they are of no great use since the dimension of Jet spaces $J^{(n)} M$ grows combinatorially with $n$. Thus the IBDP only provides a subset of the needed differential invariants to obtain a reduction. On the other hand, symmetry analysis allows to obtain a reduced equation which describes the symmetry-invariant solutions.

Discussing symmetries for PDEs requires to change slightly our notation, renouncing to the simplifications made possible by dealing only with the ODE case.

We now have $B \subseteq \mathbf{R}^{q}$ as the space for independent variables $x^{i}(i=1, \ldots, q)$; we will now write a generic vector field in $M$ as

$$
X=\xi^{i}(x, u) \frac{\partial}{\partial x^{i}}+\varphi^{a}(x, u) \frac{\partial}{\partial u^{a}}=\xi^{i} \partial_{i}+\varphi^{a} \partial_{a} .
$$

The partial derivatives of the $u^{a}$ will be denoted as $u_{J}^{a}$, with $J=\left(j_{1}, \ldots, j_{q}\right)$ a multi-index of order $|J|=j_{1}+\ldots+j_{q}$; here

$$
u_{J}^{a}:=\frac{\partial^{|J|} u^{a}}{\partial\left(x^{1}\right)^{j_{1}} \ldots \partial\left(x^{q}\right)^{j_{q}}} .
$$

We also write

$$
u_{J, i}^{a}:=\frac{\partial u_{J}^{a}}{\partial x^{i}}=\partial_{i} u_{J}^{a}
$$

For several independent variables we have correspondingly several total differential operators

$$
D_{i}=\frac{\partial}{\partial x^{i}}+u_{J, i}^{a} \frac{\partial}{\partial u_{J}^{a}}=\partial_{i}+u_{J}^{a} \partial_{a}^{J} .
$$

\footnotetext{
${ }_{\mathrm{d}}^{\mathrm{d}}$ practice, this will depend on the Lie algebraic structure of the symmetry algebra. 
Note that here and in the following we use the lazy notation

$$
\partial_{a}^{J}:=\frac{\partial}{\partial u_{J}^{a}} .
$$

The prolonged vector field $X^{(n)}$ will then be written as

$$
X^{(n)}=\xi^{i} \partial_{i}+\psi_{J}^{a} \partial_{a}^{J},
$$

where $\psi_{0}^{a}=\psi^{a}$ and the other coefficients are given by the standard prolongation formula; with our notation this is

$$
\psi_{J, i}^{a}=D_{i} \psi_{J}^{a}-u_{J, k}^{a} D_{i} \xi^{k}
$$

Remark 4. Note that the prolongation of a vector field $X$ of the general form (1.17) and of its evolutionary representative $X_{v}=Q^{a} \partial_{a}$, where now $Q^{a}=\varphi^{a}-u_{i}^{a} \xi^{i}$, are related through

$$
X_{v}^{(n)}=X^{(n)}-\xi^{k} \partial_{k}-u_{J, k}^{a} \xi^{k} \partial_{a}^{J} .
$$

Given an algebra $\mathscr{G}$ of Lie-point vector fields on $M, \mathscr{G}$-invariant solutions for $\Delta$ are solutions which are invariant under all of $\mathscr{G}$. Thus one can determine them through a symmetry reduction, which is not acting on the equation, but only on the space of solutions with the desired symmetry properties. In practice, one pass to a system of partially adapted coordinates (some of them being invariant under $\mathscr{G}$, and residual ones), and look for solutions which are expressed uniquely in terms of the invariant ones.

This can be done by complementing the system $\Delta$ with a system $\Delta_{\mathscr{G}}$ expressing the invariance condition; for an algebra with generators

$$
X_{\alpha}=\xi_{\alpha}^{i} \partial_{i}+\varphi_{\alpha}^{a} \partial_{a} \quad(\alpha=1, \ldots, r),
$$

this is written as

$$
\varphi^{a}-\xi^{i} \frac{\partial u^{a}}{\partial x^{i}}=0
$$

Remark 5. It should be noted that, albeit in general one looks for $\mathscr{G}$-invariant solutions with $\mathscr{G}$ a symmetry (sub)algebra for $\Delta$, there may exist solutions which are invariant under more general vector fields (a trivial example is often provided by the null solution); in this case one speaks of "conditional symmetries" [35] (see [14] for a generalization) or of "weak symmetries" [21,59]; the notion of "side conditions" $[57,58]$ (see also $[19,20]$ ) is also used in this context.

\section{Twisted symmetries}

Twisted symmetries were first introduced by Muriel and Romero in 2001 [40] in the form of $\lambda$ symmetries for scalar ODEs and soon extended to a more general setting [41-43]; see also [44-47, $50,52]$ for further developments; for a comprehensive review up to 2009 see [23].

Twisted symmetries are standard Lie-point vector fields in $M$ which are prolonged to vector fields in $J^{(n)} M$ through a modified ("twisted") prolongation operation, usually based on a auxiliary 
object: a function $\lambda$ for $\lambda$-symmetries, a one-form $\mu$ for $\mu$-symmetries, a matrix $\Lambda$ or $\sigma$ for $\Lambda$ or $\sigma$-symmetries. In this way we get a twisted prolongation $X_{\theta}^{(n)}=Y$; we say that $X$ is a twisted symmetry for a (partial or ordinary) differential equation $\Delta$ if this twisted prolongation satisfies the analogue of (1.3), i.e. if

$$
Y: S_{\Delta} \rightarrow \mathrm{T} S_{\Delta}
$$

Obviously this geometrical condition is also written in algebraic terms as

$$
[Y(\Delta)]_{S_{\Delta}}=0 .
$$

We will divide twisted prolongations, and hence twisted symmetries, in two classes:

(A) Those for which there is a modified prolongation operation for the single vector field; we call these "simple twisted symmetries".

(B) Those for which we consider several vector fields (generating an involution system) at once, and the modified prolongation operation acts on each vector field depending also on other vector fields; we call these "collective twisted symmetries".

Obviously one could consider the first class as a special case of the second one; however we feel that it is convenient to treat them separately. This is for several reasons: first of all because this in this way our discussion will be more clear; and then also to follow the historical development and to the benefit of the reader who is interested only in "classical" twisted symmetries (those of the first type). Moreover, as some geometrical understanding of type (A) is provided in the literature, in terms of gauge theories, it is appropriate to devote a special discussion of how this fits into the framework we are proposing here. We will discuss the gauge properties of different types of twisted symmetries, and later on how these are natural in view of the picture which emerges from our discussion.

\section{Simple twisted symmetries}

\subsection{Simple twisted symmetries: $\lambda$-symmetries}

The prototype of simple twisted symmetries is given by the $\lambda$-symmetries introduced by Muriel and Romero [40-46]; these were then generalized to $\mu$-symmetries [16, 25] (which deal also with PDEs) and to $\Lambda$ - and $\rho$-symmetries $[10,11]$.

In $\lambda$-symmetries, the prolongation operation is modified via an auxiliary smooth real function $\lambda: J^{(1)} M \rightarrow \mathbf{R}$; then (1.5) is replaced by

$$
\psi_{(k+1)}^{a}=\left(D_{x}+\lambda\right) \psi_{(k)}^{a}-u_{(k+1)}^{a}\left(D_{x}+\lambda\right) \xi .
$$

We say then that

$$
Y=X_{\lambda}^{(n)}=\xi \frac{\partial}{\partial x}+\psi_{k}^{a} \frac{\partial}{\partial u_{k}^{a}}
$$

is the $\lambda$-prolongation of $X$.

If the equation $\Delta$ is invariant under the $\lambda$-prolongation of $X$, i.e. if

$$
Y: S_{\Delta} \rightarrow \mathrm{T} S_{\Delta}
$$

we say that $\Delta$ admits $X$ as a $\lambda$-symmetry. 
Remark 6. Note that here not only the modification of the prolongation acts autonomously on each vector field, but actually each component (in the sense of the vector structure in $U$ and on the associate "derivative" spaces $U_{(k)} \subset J^{(n)} M$ for $\left.k \leq n\right)$ is transformed autonomously. It is also quite clear from (3.1) that we can interpret this as the replacement of the standard total derivative $D_{x}$ with a sort of "total covariant derivative"

$$
\nabla_{x}:=D_{x}+\lambda
$$

adopting this point of view, $\lambda$ is associated to a connection in a real line bundle over $B$, and this acts on all line bundles associated to different components of $u^{a}$ (a more precise geometrical discussion will be provided below).

Remark 7. It is also natural, in view of (3.1), to consider the case where we do not have identical connections on these different line bundles: this corresponds to considering different functions $\lambda_{a}$ for different components $\psi^{a}$. In other words we could have (with no sum on $a$ )

$$
\psi_{(k+1)}^{a}=\left(D_{x}+\lambda_{a}\right) \psi_{(k)}^{a}-u_{(k+1)}^{a}\left(D_{x}+\lambda_{a}\right) \xi .
$$

This was considered in [43], and is also a special case of Cicogna's $\Lambda$-symmetries [10-12], see Sect.3.2.

If we compare $\lambda$-prolongations and symmetries with standard prolongations and symmetries with our mind turned towards symmetry reduction of ODEs, even of a single one, a striking difference is immediately apparent: while for standard prolongations the vector field $X=(\partial / \partial u)$ has a trivial prolongation, i.e. $X^{(n)}=X$ - see the discussion in section 1.2 - now the same vector field has in general a nontrivial prolongation, due to the function $\lambda$. This means in particular that if we pass to symmetry-adapted coordinates, so that $X$ has the above form, having an equation $\Delta$ which admits $X$ as a $\lambda$-symmetry (with a nontrivial $\lambda$ ) does not entail that $\Delta$ is independent of $u$. It is thus evident that our discussion of section 1.2, based on coordinates, does not (at least directly) apply to symmetry reduction under $\lambda$-symmetries. e $^{-}$

On the other hand, it turns out that $\lambda$-prolongations possess the IBDP [42]; this shows that symmetry reduction is actually possible under $\lambda$-prolonged vector fields. As this is at the basis of the application of $\lambda$-symmetries, we will state this as a Lemma. This result is well known and, as for Lemma 1 above, we will not provide a proof (see e.g. the original proof by Muriel and Romero [42] or the discussion in [23]; see also our proof of Lemma 5 below).

Lemma 3. The IBDP holds for $\lambda$-prolonged vector fields.

The discussion in [42] provides a direct proof of the IBDP, based on analytical computations. It is also possible to proceed in a different way. In fact, it turns out that $\lambda$-prolonged vector fields are locally equivalent to ordinarily prolonged ones via a gauge transformation, and one can infer the IBDP for the $\lambda$-prolonged field from the fact it holds for the equivalent ordinarily-prolonged one. We will now discuss this point of view, starting with a discussion of the gauge equivalence [16,25] (see also [24] for detail).

\footnotetext{
${ }^{\mathrm{e}}$ Actually, there seem to be no apriori reason for a symmetry reduction under $\lambda$-symmetries to be possible. That this is the case, and actually that a kind of symmetry reduction is possible considering deformed prolongation operations, was first proved by analytical computation by Muriel and Romero.
} 
We stress beforehand that the gauge equivalence is in general only local (and not global); thus twisted symmetries are in fact more general than standard ones, even considering gauge equivalence classes.

Moreover, this local gauge equivalence is in general realized through non-local functions, i.e. functions expressed as integrals of standard smooth functions. See in this respect the discussion in Remark 10 below. $^{\mathrm{f}}$

Lemma 4. Let us consider the Lie-point vector fields $X$ and $\widetilde{X}$ on $M$, with $X$ as in (1.2) and $\widetilde{X}=\beta X$, with $\beta: M \rightarrow \mathbf{R}$ a nowhere zero smooth $\left(C^{\infty}\right)$ function on $M$. Then

$$
\beta\left(X_{\lambda}^{(n)}\right)=\widetilde{X}^{(n)},
$$

with $\lambda=\left(D_{x} \beta\right) \beta^{-1}$.

Proof. Our assumption means that

$$
\widetilde{X}=\widetilde{\xi} \partial_{x}+\widetilde{\varphi}^{a} \partial_{a} ; \tilde{\xi}=\beta \xi, \widetilde{\varphi}^{a}=\beta \varphi^{a}
$$

Let us now consider the first prolongations of these; for $\widetilde{X}^{(1)}$ we have, according to the standard prolongation formula,

$$
\begin{aligned}
\widetilde{\psi}_{(1)}^{a} & =D_{x} \widetilde{\varphi}^{a}-u_{x}^{a} D_{x} \widetilde{\xi}=D_{x}\left(\beta \varphi^{a}\right)-u_{x}^{a} D_{x}(\beta \xi) \\
& =\beta\left(D_{x} \varphi^{a}-u_{x}^{a} D_{x} \xi\right)+\left(D_{x} \beta\right)\left(\varphi^{a}-u_{x}^{a} \xi\right) \\
& =\beta \psi_{(1)}^{a}+\left(D_{x} \beta\right)\left(\varphi^{a}-u_{x}^{a} \xi\right) \\
& =\beta\left[\psi_{(1)}^{a}+\beta^{-1}\left(D_{x} \beta\right)\left(\varphi^{a}-u_{x}^{a} \xi\right)\right] .
\end{aligned}
$$

It is easy to check that this holds for prolongations in $J^{(n)} M$, for whatever $n$. In fact, suppose it holds for $k$; then proceeding exactly as above we have

$$
\begin{aligned}
\widetilde{\psi}_{(k+1)}^{a} & =D_{x} \widetilde{\psi}_{(k)}^{a}-u_{(k+1)}^{a} D_{x} \widetilde{\xi}=D_{x}\left(\beta \psi_{(k)}^{a}\right)-u_{(k+1)}^{a} D_{x}(\beta \xi) \\
& =\beta\left[\psi_{(k+1)}^{a}+\beta^{-1}\left(D_{x} \beta\right)\left(\psi_{(k)}^{a}-u_{(k+1)}^{a} \xi\right)\right] .
\end{aligned}
$$

As we have just checked the required property holds for $k=0$, the proof is completed by recursion up to any desired degree.

Remark 8. We have thus seen that the multiplication by $\beta$ connects $X$ and $\widetilde{X}$ at the level of vector fields in $M$, while at the level of vector fields in $J^{(n)} M$ it connects the $\lambda$-prolongation of $X$ (with $\left.\lambda=\left(D_{x} \beta\right) \beta^{-1}\right)$ and the standard prolongation of $\widetilde{X}$. As by assumption $\beta \neq 0$ in all points of $M$, the vector fields $X$ and $\widetilde{X}$ are collinear, and we have seen that the $\lambda$-prolongation of a vector field $X$ is collinear (through a factor $\beta$ ) to the standard prolongation of a collinear (through the same factor

\footnotetext{
${ }^{\mathrm{f}}$ As these integrals appear as arguments of an exponential function, and to avoid confusion with locality in the sense just mentioned above, we will often speak of "exponential type" functions or "exponential vector fields"; this follows the notation suggested by Muriel and Romero [40,45], who in turn refer to Olver [55] for the original definition.
} 
$\beta$ ) vector field $\widetilde{X}$. We can summarize these relations in the form of a commutative diagram:

$$
\begin{gathered}
X \stackrel{\beta}{\longrightarrow} \widetilde{X} \\
\underset{\perp}{\lambda-\text { prol }} \underset{\mid}{\mid \text { prol }} \\
X_{\lambda}^{(n)} \stackrel{\beta}{\longrightarrow} \widetilde{X}_{0}^{(n)}
\end{gathered}
$$

The functions $\lambda$ and $\beta$ are related by

$$
\lambda=\left(D_{x} \beta\right) \beta^{-1}=D_{x}(\log \beta) ;
$$

we can also write

$$
\beta=\exp \left[\int \lambda d x\right]
$$

Note also that as $\beta: M \rightarrow \mathbf{R}$, it results $\lambda: J^{1} M \rightarrow \mathbf{R}$; the $C^{\infty}$ smoothness of $\beta$ entails $C^{\infty}$ smoothness of $\lambda$.

Remark 9. The effect of multiplication of a $\lambda$-prolonged vector field $X$ by a smooth function $f$ has been considered in [40]; in particular, Lemma 5.1 in there shows that in this way one can obtain a new $\lambda$-prolonged vector field with a new function $\bar{\lambda}=\lambda-[X(f) / f]$; choosing $X(f) / f=\lambda$ one obtains $\bar{\lambda}=0$.

Remark 10. The functions $\lambda\left(x, u, u_{x}\right)$ and $\beta(x, u)$ are related through (3.5). If $\beta$ is given, this straightforwardly defines $\lambda$. On the other hand, if $\lambda$ is given and we want to determine $\beta$, the solution is given by (3.6), but here it is in general impossible to write $\beta$ as a local function, and it can only be expressed as an integral; we also say that it is a non-local function (note no confusion should be made with locality in the sense of the domain of definition of a function). If $X=\xi \partial_{x}+\varphi^{a} \partial_{a}$, we will get

$$
\widetilde{X}=e^{\int \lambda d x}\left[\xi \partial+\varphi^{a} \partial_{a}\right]
$$

i.e. an "exponential vector field" in the sense of Olver [55], see also [27,40,45]. ${ }^{\mathrm{g}}$

\subsection{Simple twisted symmetries: $\mu$-symmetries}

A substantial generalization of $\lambda$-symmetries concerns the possibility of defining twisted prolongation operators also for $\mathrm{PDEs}^{\mathrm{h}}$; discussing this frame requires to use the general notation (see subsection 1.5).

\footnotetext{
$\overline{{ }^{g}}$ Note also that the primitive of a function $\lambda$, hence $\beta$, is not uniquely defined; however adding a constant term to $\beta$ would give a global gauge transformation, not relevant here.

hThe name "twisted" gets its justification in this framework, as we will see in a moment.
} 
Now the role of the function $\lambda$ is taken by a semibasic one-form $\mu \in \Lambda^{1}\left(J^{1} M\right)$, which we write as

$$
\mu=\Lambda_{i} \mathrm{~d} x^{i}
$$

the $\Lambda_{i}$ are matrix functions, $\Lambda: J^{1} M \rightarrow \operatorname{Mat}(\mathbf{R}, q)$. The form $\mu$ should be such that $\mathrm{d} \mu$ belongs to the Cartan ideal $\mathscr{C}[26,61,64]$ generated by the contact forms in $J^{n} M$, i.e.

$$
\mathrm{d} \mu \in \mathscr{C} .
$$

This amounts to the requirement that $\mu$ should satisfy the horizontal Maurer-Cartan equation

$$
D \mu+\frac{1}{2}[\mu, \mu]=0 .
$$

If seen in terms of the matrices $\Lambda_{i}$, this is the compatibility condition (here the commutator is the standard commutator between matrices)

$$
D_{i} \Lambda_{j}-D_{j} \Lambda_{i}+\left[\Lambda_{i}, \Lambda_{j}\right]=0 .
$$

We note that defining the "total covariant derivatives"

$$
\nabla_{i}:=D_{i}+\Lambda_{i}
$$

the previous formulas can be rewritten as the zero-curvature condition

$$
\left[\nabla_{i}, \nabla_{j}\right]=0 .
$$

The condition (3.9) - or equivalently (3.10), or (3.12) - guarantee that $\mu$ is horizontally closed ${ }^{\mathrm{i}}$ :

$$
D \mu:=\left(D_{i} \Lambda_{j}\right) \mathrm{d} x^{i} \wedge \mathrm{d} x^{j}=0 .
$$

This in turn is nothing else than a restatement of (3.8).

We now define recursively the $\mu$-prolongation of the vector field (1.17) as

$$
Y=X_{\mu}^{(n)}=\xi^{i} \frac{\partial}{\partial x^{i}}+\psi_{(J)}^{a} \frac{\partial}{\partial u_{(J)}^{a}}=\xi^{i} \partial_{i}+\psi_{(J)}^{a} \partial_{a}^{J},
$$

with $\psi_{(0)}^{a}=\varphi^{a}$ and

$$
\psi_{(J, i)}^{a}=\left(\delta_{b}^{a} D_{i}+\left(\Lambda_{i}\right)_{b}^{a}\right) \varphi^{b}-u_{J, k}^{b}\left(\delta_{b}^{a} D_{i}+\left(\Lambda_{i}\right)_{b}^{a}\right) \xi^{k} .
$$

Remark 11. It should be noted that $\mu$-symmetries apply both for (systems of) ODEs and (systems of) PDEs; in the first case we actually have a single matrix $\Lambda$ (and no compatibility condition, so that this is in several sense a degenerate case), and one also uses the name of $\Lambda$-symmetries $[10,11]$. These will be specially relevant in the following, considering reduction of systems of ODEs.

We will start by discussing the IBDP for $\mu$-symmetries. Here it happens that the IBDP does in general not hold for $\mu$-prolonged vector fields. If the matrices $\Lambda_{i}$ are multiples of the identity, then

${ }^{\text {i }}$ The appearance of total (hence horizontal) derivatives is related to taking into account the contact structure in $J^{n} M$; see $[16,25]$ for details. 
G. Gaeta

the IBDP property holds; the proof of this fact reduces to a variant of the proofs of Lemma 1 and Lemma 3.

Lemma 5. Let $\mu=\Lambda_{i} \mathrm{~d} x^{i}$, the matrices $\Lambda_{i}$ being multiple of the identity,

$$
\left(\Lambda_{i}\right)_{b}^{a}=\lambda_{i} \delta_{b}^{a}
$$

with $\lambda_{i}: J^{1} M \rightarrow \mathbf{R}$ scalar $C^{\infty}$ functions. The IBDP holds for such $\mu$-prolonged vector fields.

Proof. We will proceed as in the standard proof of Lemma 1 (see [55]); we will however use the more general notation (so to include also the case of PDEs - albeit, as mentioned above, the IBDP property is not so useful in this context). We thus have

$$
D_{i}=\partial_{i}+u_{(J, i)}^{a} \partial_{a}^{J}, \quad Y=\xi^{i} \partial_{i}+\psi_{(J)}^{a} \partial_{a}^{J} ;
$$

it follows that

$$
\left[Y, D_{i}\right]=\left[\psi_{(J, i)}^{a}-\left(D_{i} \psi_{(J)}^{a}\right)\right] \partial_{a}^{J}-\left(D_{i} \xi^{k}\right) \partial_{k} .
$$

If $Y$ is a $\mu$-prolongation, it follows from (3.15) that (adding and subtracting a factor proportional to $\xi^{k} \partial_{k}$ through a function $\rho_{i}$ ) this reads

$$
\begin{aligned}
{\left[Y, D_{i}\right] } & =\left[\left(\Lambda_{i}\right)_{b}^{a}\left(\psi_{J}^{b}-u_{J, k}^{b} \xi^{k}\right)-u_{(J, k)}^{a}\left(D_{i} \xi^{k}\right)\right] \partial_{a}^{J}-\left(D_{i} \xi^{k}\right) \partial_{k} \\
& =\left[\left(\Lambda_{i}\right)_{b}^{a}\left(\psi_{J}^{b}-u_{J, k}^{b} \xi^{k}\right)\right] \partial_{a}^{J}-\left(D_{i} \xi^{k}\right)\left[\partial_{k}+u_{(J, k)}^{a} \partial_{a}^{J}\right] \\
& =\left[\left(\Lambda_{i}\right)_{b}^{a}\left(\psi_{J}^{b}-u_{J, k}^{b} \xi^{k}\right)\right] \partial_{a}^{J}-\left(D_{i} \xi^{k}\right) D_{k} \\
& =\left[\rho_{i} \xi^{k} \partial_{k}+\left(\Lambda_{i}\right)_{b}^{a} \psi_{J}^{b} \partial_{a}^{J}\right]-\xi^{k}\left[\rho_{i} \partial_{k}+\left(\Lambda_{i}\right)_{b}^{a} u_{(J, k)}^{b} \partial_{a}^{J}\right]-\left(D_{i} \xi^{k}\right) D_{k} ;
\end{aligned}
$$

note that here the functions $\rho_{i}$ are arbitrary ones. If in addition the matrices $\Lambda_{i}$ are multiples of the identity, see (3.16), this reads

$$
\left[Y, D_{i}\right]=\left[\rho_{i} \xi^{k} \partial_{k}+\lambda_{i} \psi_{J}^{a} \partial_{a}^{J}\right]-\xi^{k}\left[\rho_{i} \partial_{k}+\lambda_{i} u_{(J, k)}^{a} \partial_{a}^{J}\right]-\left(D_{i} \xi^{k}\right) D_{k} .
$$

Finally, we make use of our freedom in choosing the functions $\rho_{i}$ to set $\rho_{i}=\lambda_{i}$, and obtain

$$
\begin{aligned}
{\left[Y, D_{i}\right] } & =\lambda_{i}\left[\xi^{k} \partial_{k}+\psi_{J}^{a} \partial_{a}^{J}\right]-\lambda_{i} \xi^{k}\left[\partial_{k}+u_{(J, k)}^{a} \partial_{a}^{J}\right]-\left(D_{i} \xi^{k}\right) D_{k} \\
& =\lambda_{i} Y-\left[\lambda_{i} \xi^{k}+\left(D_{i} \xi^{k}\right)\right] D_{k} .
\end{aligned}
$$

Now let us consider the IBDP; we want to prove that if $\eta$ and $\zeta_{(m)}$ are differential invariants for $Y$, so is $\zeta_{(m+1)}:=\left(D_{i} \zeta_{(m)}\right) /\left(D_{i} \eta\right)$, for any $i=1, \ldots, p$. We do of course have

$$
Y\left[\frac{D_{i} \zeta_{(m)}}{D_{i} \eta}\right]=\frac{Y\left(D_{i} \zeta_{(m)}\right) \cdot\left(D_{i} \eta\right)-\left(D_{i} \zeta_{(m)}\right) \cdot Y\left(D_{i} \eta\right)}{\left(D_{i} \eta\right)^{2}}
$$

so that we have to prove that

$$
Y\left(D_{i} \zeta_{(m)}\right) \cdot\left(D_{i} \eta\right)=\left(D_{i} \zeta_{(m)}\right) \cdot Y\left(D_{i} \eta\right)
$$

This can be rewritten as

$$
\begin{gathered}
{\left[Y, D_{i}\right]\left(\zeta_{(m)}\right) \cdot\left(D_{i} \eta\right)+D_{i}\left[Y\left(\zeta_{(m)}\right)\right]=\left(D_{i} \zeta_{(m)}\right) \cdot\left[Y, D_{i}\right](\eta)+D_{i}[Y(\eta)]} \\
\text { Co-published by Atlantis Press and Taylor \& Francis } \\
\text { Copyright: the authors } \\
606
\end{gathered}
$$


since by assumption $Y\left(\zeta_{(m)}\right)=0=\mathscr{Y}(\eta)$, this reduces in turn to

$$
\left[Y, D_{i}\right]\left(\zeta_{(m)}\right) \cdot\left(D_{i} \eta\right)=\left(D_{i} \zeta_{(m)}\right) \cdot\left[Y, D_{i}\right](\eta) .
$$

We can now make use of (3.17), and rewrite this as

$$
\begin{aligned}
& \lambda_{i} Y\left(\zeta_{(m)}\right) \cdot\left(D_{i} \eta\right)-\left(\lambda_{i} \xi^{k}+D_{i} \xi^{k}\right) D_{k}\left(\zeta_{(m)}\right) \cdot\left(D_{i} \eta\right) \\
& =\lambda_{i}\left(D_{i} \zeta_{(m)}\right) \cdot Y(\eta)-D_{i}\left(\zeta_{(m)}\right) \cdot\left(\lambda_{i} \xi^{k}+D_{i} \xi^{k}\right)\left(D_{k} \eta\right)
\end{aligned}
$$

recalling again $Y\left(\zeta_{(m)}\right)=0=\mathscr{Y}(\eta)$, we are reduced to the identity

$$
\left(\lambda_{i} \xi^{k}+D_{i} \xi^{k}\right) \cdot D_{k}\left(\zeta_{(m)}\right) \cdot\left(D_{i} \eta\right)=D_{i}\left(\zeta_{(m)}\right) \cdot\left(\lambda_{i} \xi^{k}+D_{i} \xi^{k}\right) \cdot\left(D_{k} \eta\right) .
$$

This is trivially true, and the proof is completed.

Remark 12. This proof also provides a proof of Lemma 1 and Lemma 3; in fact Lemma 1 is obtained setting all the $\Lambda_{i}$ (i.e. the $\lambda_{i}$ ) to zero, and Lemma 3 restricting to the one-dimensional case.

Remark 13. The $\mu$-prolonged vector fields with $\mu$ as in (3.16) are also called $\Lambda$-prolonged (to emphasize the similarity with standard $\lambda$-prolongations). Note that, as immediately apparent from the proof (and as can be checked by simple examples) the IBDP does not hold for general $\mu$ prolonged vector fields, i.e. when (3.16) is not satisfied.

Note also that the proof of IBDP for $\Lambda$-prolongations seems to appear in the literature only by considering the evolutionary representative of the vector field $X$ and its $\mu$-prolonged corresponding vector field; in this respect, see also Sect.3.3 below.

It turns out that $\mu$-prolonged evolutionary vector fields are locally equivalent to ordinarily prolonged ones via a gauge transformation (and one can infer the IBDP for the $\Lambda$-prolonged field from the fact it holds for the equivalent ordinarily-prolonged one). We will now discuss this point of view, starting with a discussion of the gauge equivalence (see also [16,24] for detail); we stress this only holds for vector fields in evolutionary form, see also Sect.3.3.

Lemma 6. Let us consider the evolutionary vector fields $X$ and $\widetilde{X}$ on $M$, with

$$
X=Q^{a} \partial_{a}, \tilde{X}=\left(A_{b}^{a} Q^{b}\right) \partial_{a},
$$

with $A: M \rightarrow \operatorname{Mat}(\mathbf{R}, q)$ a nowhere zero smooth $\left(C^{\infty}\right)$ matrix function on $M$. Then

$$
A\left(X_{\mu}^{(n)}\right)=\widetilde{X}^{(n)},
$$

with $\mu=(D A) A^{-1}$.

Proof. First of all we note that (3.18) should be meant as a shorthand notation for

$$
X_{\mu}^{(n)}=Q_{J}^{a} \partial_{a}^{J}, \widetilde{X}^{(n)}=\widetilde{Q}_{J}^{a} \partial_{a}^{J}
$$

with $Q$ and $\widetilde{Q}$ related through

$$
A_{b}^{a} Q_{J}^{a}=\widetilde{Q}_{J}^{a}
$$

and of course $Q_{J}^{a}$ obeying the $\mu$-prolongation formula and $\widetilde{Q}_{J}^{a}$ the standard one, so that

$$
\begin{gathered}
Q_{J, i}^{a}=D_{i} Q_{J}^{a}+\left(\Lambda_{i}\right)_{b}^{a} Q_{J}^{b}, \widetilde{Q}_{J, i}^{a}=D_{i} \widetilde{Q}_{J}^{q} . \\
\text { Co-published by Atlantis Press and Taylor \& Francis } \\
\text { Copyright: the authors } \\
607
\end{gathered}
$$


Let us write, for all $J$,

$$
Q_{J}^{a}=A_{b}^{a} P_{J}^{b},
$$

with $A$ a $C^{\infty}$ and nowhere singular matrix function on $M$. Then the $\mu$-prolongation formula requires $Q_{J, i}^{a}=D_{i}\left(Q_{J}^{a}\right)+\left(\Lambda_{i}\right)_{b}^{a} Q_{J}^{b}=D_{i}\left(A_{b}^{a} P_{J}^{b}\right)+\left(\Lambda_{i}\right)_{b}^{a} A_{c}^{b} P_{J}^{c}=D_{i}\left(A_{b}^{a}\right) P_{J}^{b}+A_{b}^{a} D_{i}\left(P_{J}^{b}\right)+\left(\Lambda_{i} A\right)_{b}^{a} P_{J}^{b}$.

On the other hand, we know that

$$
Q_{J, i}^{a}=A_{b}^{a} P_{J, i}^{b}
$$

comparing these two formulas, we get

$$
P_{J, i}^{a}=D_{i} P_{J}^{a}+\left[A^{-1} D_{i}(A)\right]_{b}^{a} P_{J}^{b}+\left(A^{-1} \Lambda_{i} A\right)_{b}^{a} P_{J}^{b} .
$$

We conclude that the $P_{J}^{a}$ satisfy the standard prolongation formula provided $A$ satisfies

$$
A^{-1}\left(D_{i} A\right)+A^{-1} \Lambda_{i} A=0 ;
$$

equivalently, provided

$$
\left(D_{i} A\right) A^{-1}=\Lambda_{i}
$$

As $D A=\left(D_{i} A\right) \mathrm{d} x^{i}$, the proof is completed.

Remark 14. We have thus seen that the action of $A$ connects $X$ and $\widetilde{X}$ at the level of (evolutionary) vector fields in $M$, while at the level of vector fields in $J^{(n)} M$ it connects the $\mu$-prolongation of $X$ (with $\mu=(D A) A^{-1}$ ) and the standard prolongation of $\widetilde{X}$. We can summarize these relations in the form of a commutative diagram:

$$
\begin{gathered}
X \stackrel{A}{\longrightarrow} \widetilde{X} \\
\underset{\mid}{\mid \mu-\text { prol }} \underset{\mid \text { prol }}{ } \\
X_{\lambda}^{(n)} \stackrel{A}{\longrightarrow} \widetilde{X}_{0}^{(n)}
\end{gathered}
$$

The matrices $\Lambda_{i}$ defining $\mu$ and $A$ are related by

$$
\Lambda_{i}=\left(D_{i} A\right) A^{-1}=D_{i}(\log A) ;
$$

we can also write

$$
A=\exp \left[\int \Lambda_{i} d x^{i}\right] .
$$

Note also that $\Lambda_{i}: J^{1} M \rightarrow \operatorname{Mat}(\mathbf{R}, q)$; the $C^{\infty}$ smoothness of $A$ entails $C^{\infty}$ smoothness of the $\Lambda_{i}$. $\odot$

Remark 15. In the case $\Lambda_{i}=\lambda_{i} I$, the equivalence is through a simple rescaling of the vector fields. $\odot$

Remark 16. The discussion of Remark 10 also applies here. That is, the relation between $A$ and the $\Lambda_{i}$ is described by (3.20), but this can be read in two ways: if $A$ is given, we immediately obtain the $\Lambda_{i}$; on the other hand, if the $\Lambda_{i}$ are assigned and we look for the corresponding $A$, we obtain (3.21), 
but it is in general impossible to express $A$ as a local function, and we are again led to consider exponential type vector fields $[40,45,55]$.

When we look for invariant solutions to PDEs, we focus on the invariant sections of $\left(J^{n} M, \pi_{n}, B\right)$. It happens that the invariant sets for the standard prolongation of a vector field and for its $\mu$ prolongation do coincide. In fact, we have more precisely the following results.

Lemma 7. Let $X$ be an evolutionary vector field of the form $X=Q^{a} \partial_{a}$. Denote by $X_{\mu}^{(n)}$ its standard prolongation with $\mu=\Lambda_{i} \mathrm{~d} x^{i}$, and by $X^{(n)}=X_{0}^{(n)}$ its ordinary prolongation; write

$$
X_{\mu}^{(n)}=\Psi_{J}^{a} \partial_{a}^{J} ; X^{(n)}=\Phi_{J}^{a} \partial_{a}^{J} .
$$

Then the coefficients $\Psi_{J}^{a}$ (obeying the $\mu$-prolongation formula) and $\Phi_{J}^{a}$ (obeying the standard prolongation formula) are related by

$$
\Psi_{J}^{a}=\Phi_{J}^{a}+F_{J}^{a}
$$

the difference term satisfies $F_{0}^{a}=0$ and obeys the recursion relation

$$
F_{J, i}^{a}=\left[\delta_{b}^{a} D_{i}+\left(\Lambda_{i}\right)_{b}^{a}\right] F_{J}^{b}+\left(\Lambda_{i}\right)_{b}^{a}\left(D_{J} Q^{b}\right) .
$$

Proof. This is Theorem 6 of [25]; see there for the detailed proof (which amounts to a rather involved explicit computation).

Remark 17. The $X$-invariant sections $\gamma$ of $\left(M, \pi_{0}, B\right)$ are characterized by the vanishing of $Q$ on them (or more precisely on their first prolongation $\gamma^{(1)}$ ). It follows from Lemma 7 that on the subset of sections with $Q=0$ we have $F_{J}^{a}=0$ at all orders (recall $F_{0}^{a}=0$ ). Thus we conclude that standard and $\mu$-prolongations of evolutionary vector fields do coincide on the set of invariant sections, hence on invariant functions.

Remark 18. As recalled in Remark 5 above, a particularly effective generalization of the standard symmetry approach to determining invariant solutions to PDEs is to consider "weak" or "conditional" symmetries [21,35, 57-59]. The idea is to look for transformations which are not proper symmetries of the PDEs under study (thus do not in generalmap solutions into solutions) but such that the PDEs admit solutions which are invariant under these transformations. One could wonder if an extension of this concept to the realm of twisted symmetries - i.e. considering weak or conditional twisted symmetries - is possible and effective. The previous Lemma and Remark show that this is possible but not effective, in that on the set of invariant sections we would reduce to consider standard prolongations and hence standard weak or conditional symmetries. The situation is different when considering so called "partial symmetries": these are transformations which map a subset of solution into other solutions of the same subset, and in the non trivial case the solutions will be mapped into different solutions. In this case the extension of the approach to twisted symmetries can give nontrivial results, as discussed in [20].

Remark 19. Here we are not discussing the relation of $\mu$-prolongations and symmetries with $\mu$ related deformed exterior and Lie derivatives; this point of view was advocated by Morando [37]. 


\section{3. $\mu$-symmetries without evolutionary representatives}

We have obtained our result about gauge equivalence of $\mu$-prolonged vector fields with standardly prolonged ones at the price of dealing exclusively with vertical vector fields; if the vector fields we wish to consider are not vertical, we can always resort to their evolutionary representative in order to be within this framework. It should be noted, however, that when $X=Q^{a} \partial_{a}$ is the evolutionary representative of a vector field $X_{0}$ with a horizontal component, say $X_{0}=\xi^{i} \partial_{i}+\varphi^{a} \partial_{a}$ (we will assume at least one of the $\xi^{i}$ is not identically zero), we are not at all guaranteed that the vertical vector field $\widetilde{X}$ whose standard prolongation is gauge-equivalent to $Y=X_{\lambda}^{(n)}$ is the evolutionary representative of some Lie-point vector field $\widetilde{X}_{0}$ in $M$. This point is usually overlooked in the literature about $\mu$-prolongations and $\mu$-symmetries, and we want to discuss it in some detail (it will suffice to consider first prolongations, i.e. $n=1)$.

For $X_{0}$ as above, we will have $Q^{a}=\varphi^{a}-u_{i}^{a} \xi^{i}$; thus

$$
Y=Q^{a} \partial_{a}+\left[\left(D_{i} Q^{a}\right)+\left(\Lambda_{i}\right)_{b}^{a} Q^{b}\right] \partial_{a}^{i} .
$$

We will look for $\widetilde{X}_{0}$ in the form

$$
\widetilde{X}_{0}=\eta^{i} \partial_{i}+\vartheta^{a} \partial_{a}
$$

its evolutionary representative is

$$
\widetilde{X}=P^{a} \partial_{a}=\left(\vartheta^{a}-u_{j}^{a} \eta^{j}\right) \partial_{a} .
$$

The standard prolongation of this is

$$
Z=P^{a} \partial_{a}+\left(D_{i} P^{a}\right) \partial_{a}^{i}=\left(\vartheta^{a}-u_{i}^{a} \eta^{i}\right)+\left[\left(D_{i} \vartheta^{a}\right)-u_{j, i}^{a} \eta^{j}-u_{j}^{a}\left(D_{i} \eta^{j}\right)\right] \partial_{a}^{i} .
$$

We note that dependence on $u_{i}^{a}$ is explicit in both $P^{a}$ and $Q^{a}$, as $\xi^{i}, \eta^{i}, \varphi^{a}, \vartheta^{a}$ only depend on $x$ and $u$; thus requiring that $\widetilde{X}$ be the gauge transform via a map $A$ of $X$, i.e. that $Q^{a}=A_{b}^{a} P^{b}$ amounts to the requirements

$$
\begin{aligned}
\varphi^{a} & =A_{b}^{a} \vartheta^{b}, \\
u_{j}^{a} \xi^{j} & =A_{b}^{a} u_{i}^{b} \eta^{i} .
\end{aligned}
$$

Note that we should also require the same holds for $Y$ and $Z$; this would fix the relation between $A$ and the $\Lambda_{i}$ as discussed above. We will thus suppose $A$ is known.

Now, it is clear that if we know $A$ then (3.23) immediately provides

$$
\vartheta^{a}=\left(A^{-1}\right)_{b}^{a} \varphi^{b} .
$$

On the other hand, (3.24) does not provide $\eta^{i}$ with equal ease. First of all, we note that albeit we have sums on the indices $i$ (in the right hand side) and $j$ (in the left hand side), these indices are not acted upon by the map $A$, so that the equality must hold for any index, and we can write

$$
u_{i}^{a} \xi^{i}=\sum_{b} A_{b}^{a} u_{i}^{b} \eta^{i}
$$

with no sum on $i$. This is a system of equations (indexed by $a$ ), which should hold for any value of the $u_{i}^{a}$; thus (unless the $\xi^{i}$ are all zero) it may be satisfied only if $A$ is diagonal. But if $A$ is 
diagonal, then $\Lambda_{i}=\left(D_{i} A\right) A^{-1}$ is also diagonal. We conclude that a necessary condition for $\widetilde{X}$ to be the evolutionary representative of a vector field $\widetilde{X}_{0}$ is that the $\Lambda_{i}$ (and $A$ ) are diagonal.

If this is the case, say with $A=\operatorname{diag}\left(\alpha_{1}, \ldots, \alpha_{p}\right)$, then (3.24) splits into $p$ scalar equations (for $b=1, \ldots, p)$,

$$
\xi^{i}=\alpha_{b} \eta^{i}
$$

it suffices to recall we have assumed $A$ is invertible, and hence all the $\alpha_{k}$ are nonzero, to conclude that in this case

$$
\eta^{i}=\left(\alpha_{b}\right)^{-1} \xi^{i}
$$

Note that unless $\alpha_{1}=\ldots=\alpha_{p}$, the equations for different $b$ would give different determinations of the $\eta^{i}$; thus $\eta^{i}$ (and hence the vector field $\widetilde{X}_{0}$ ) can be determined only for $A$ a multiple of the identity, $A=\lambda I$.

Our discussion can also be conducted in a slightly different way (reaching of course the same conclusions). That is, in order to reconstruct the original Lie-point vector field $X$ from its evolutionary representative $X_{v}$ it suffices, in view of the explicit expression for $Q^{a}=\varphi^{a}-u_{i}^{a} \xi^{i}$ and of the fact $\varphi$ and $\xi$ do not depend on $u$ derivatives, to write (no sum on $a$ )

$$
\xi^{i}=-\frac{\partial Q^{a}}{\partial u_{i}^{a}} ; \varphi^{a}=Q^{a}+u_{i}^{a} \frac{\partial Q^{a}}{\partial u_{i}^{a}}
$$

Note that we do not sum on $a$ in the formula for $\xi^{i}$; we have instead that the result is the same for any $a$.

When considering $\widetilde{X}=P^{a} \partial_{a}$ with $P=A^{-1} Q$, as seen above, if we want to write $P$ as $P^{a}=$ $\vartheta^{a}-u_{i}^{a} \eta^{i}$ we run in troubles in the determination of $\eta^{i}$. In fact, we immediately have

$$
P^{a}=\left(A^{-1}\right)_{b}^{a} \varphi^{b}-\left[\left(A^{-1}\right)_{b}^{a} u_{i}^{b}\right] \xi^{i}
$$

According to the prescription (3.27), we immediately obtain

$$
\vartheta^{a}=\left(A^{-1}\right)_{b}^{a} \varphi^{b}
$$

as it should be. But for what concerns $\eta$, the same (3.27) would provide (again, no sum on $a$ )

$$
\eta^{i}=\frac{\partial P^{a}}{\partial u_{i}^{a}}=\left(A^{-1}\right)_{a}^{a} \xi^{i}
$$

This would provide different results for different choices of $a$; unless of course all the diagonal element of $A^{-1}$ are the same.

Summarizing, if we have vector fields with horizontal components, then the diagram (3.19) applies to the evolutionary representative, and $\widetilde{X}$ will in general be a generalized vector field which is not the evolutionary representative of a Lie-point vector field $\widetilde{X}_{0}$ in $M$; and this unless $A$ is diagonal (which in turn requires the $\Lambda_{i}$ are all diagonal). In this case, $\widetilde{X}_{0}$ is identified by (3.22) with (3.25) and (3.26). 


\section{Collective and combined twisted symmetries}

\subsection{Collective twisted symmetries: $\sigma$-symmetries}

So far we have considered vector fields with modified prolongation rule, but each vector field was however prolonged independently. It is possible to consider a different modification of the prolongation operation, which makes sense on sets of vector fields; these must be in involution (in Frobenius sense).

It should be noted that so far these have been studied (and shown to be useful) only in the context of ODEs; we will thus restrict to this setting, i.e. have only one independent variable, denoted with $x$.

We will thus consider a set of vector fields $X_{\alpha}(\alpha=1, \ldots, r)$ on $M$, written in coordinates as

$$
X_{\alpha}=\xi_{\alpha} \frac{\partial}{\partial x}+\varphi_{\alpha}^{a} \frac{\partial}{\partial u^{a}}
$$

and satisfying the involution relations

$$
\left[X_{\alpha}, X_{\beta}\right]=f_{\alpha \beta}^{\gamma} X_{\gamma}
$$

where the $f_{\alpha \beta}^{\gamma}: M \rightarrow \mathbf{R}$ are smooth functions. Thus we deal with a Lie module of vector fields in $M$.

We will write the $\sigma$-prolongations of these - to be characterized by the following formula (4.4) - as

$$
Y_{\alpha}=\xi_{\alpha} \frac{\partial}{\partial x}+\psi_{\alpha,(k)}^{a} \frac{\partial}{\partial u_{(k)}^{a}}
$$

note that the index $k$ refers to the order of derivations.

Here $\psi_{\alpha, 0}^{a}=\varphi_{\alpha}^{a}$, and the $\psi_{\alpha,(k)}^{\alpha}$ satisfy the $\sigma$-prolongation formula

$$
\psi_{\alpha,(k+1)}^{a}=\left(D_{x} \psi_{\alpha,(k)}^{a}-u_{(k+1)}^{a} D_{x} \xi_{\alpha}\right)+\sigma_{\alpha}^{\beta}\left(\psi_{\beta,(k)}^{a}-u_{(k+1)}^{a} \xi_{\beta}\right)
$$

here the $\sigma_{\alpha}^{\beta}$ are smooth real functions on $J^{1} M$.

We stress that while $\mu$-prolongations were mixing different components of the same vector field, the $\sigma$-prolongations mix the same component (vector index $a$ ) of different vector fields. In particular, this implies that $\sigma$-prolongations make sense for sets (actually, as noted above, Lie modules) of vector fields. ${ }^{\mathrm{j}}$

Lemma 8. The $\sigma$-prolonged vector fields satisfy

$$
\left[Y_{\alpha}, D_{x}\right]=\sigma_{\alpha}^{\beta} Y_{\beta}-\left(D_{x} \xi_{\alpha}+\sigma_{\alpha}^{\beta} \xi_{\beta}\right) D_{x}
$$

Conversely, if the vector fields $Y_{\alpha}$ satisfy (4.5), they are $\sigma$-prolonged.

${ }^{\mathrm{j}}$ In the case of a trivial module, generated by a single vector field, we are reduced to $\lambda$-prolongations. 
Proof. This follows by a simple computation, making use of (4.4). In fact, with the notation introduced earlier on, we have

$$
\begin{aligned}
{\left[Y_{\alpha}, D_{x}\right] } & =\left(\psi_{\alpha,(k+1)}^{a}-D_{x} \psi_{\alpha,(k)}^{a}\right) \partial_{a}^{k}-\left(D_{x} \xi_{\alpha}\right) \partial_{x} \\
& =\left(-u_{(k+1)}^{a}\left(D_{x} \xi_{\alpha}\right)+\sigma_{\alpha}^{\beta}\left(\psi_{\beta,(k)}^{a}-u_{(k+1)}^{a}\right)\right) \partial_{a}^{k}-\left(D_{x} \xi_{\alpha}\right) \partial_{x} \\
& =-\left(D_{x} \xi_{\alpha}\right) D_{x}+\sigma_{\alpha}^{\beta}\left(Y_{\beta}-\xi_{\beta} \partial_{x}\right)-\sigma_{\alpha}{ }^{\beta} \xi_{\beta}\left(D_{x}-\partial_{x}\right) \\
& =-\left(\left(D_{x} \xi_{\alpha}\right)+\sigma_{\alpha}{ }^{\beta} \xi_{\beta}\right) D_{x}+\sigma_{\alpha}{ }^{\beta} Y_{\beta} .
\end{aligned}
$$

The converse statement follows performing the computation in reverse order.

Remark 20. It should be stressed that for a generic choice of $\sigma_{\alpha}^{\beta}$, the $\sigma$-prolonged vector fields $Y_{\alpha}$ would not satisfy the same involution relations as the $X_{\alpha}$. It can be shown (see Theorem 2 in [17]; the proof is based on a relatively long computation) that this is the case if and only if $\sigma$ satisfies

$$
\left[\left(Y_{\alpha}\left(\sigma_{\beta}^{\gamma}\right)-Y_{\beta}\left(\sigma_{\alpha}^{\gamma}\right)\right)+\left(\left(D_{x} f_{\alpha \beta}^{\gamma}\right)+\sigma_{\alpha}^{\eta} f_{\eta \beta}^{\gamma}-\sigma_{\beta}^{\eta} f_{\eta \alpha}^{\gamma}-f_{\alpha \beta}^{\eta} \sigma_{\eta}^{\gamma}\right)\right] \varphi_{\gamma}^{a}=0
$$

a sufficient (but not necessary) for this to hold is to have

$$
\left(Y_{\alpha}\left(\sigma_{\beta}^{\gamma}\right)-Y_{\beta}\left(\sigma_{\alpha}^{\gamma}\right)\right)+\left(\left(D_{x} f_{\alpha \beta}^{\gamma}\right)+\sigma_{\alpha}^{\eta} f_{\eta \beta}^{\gamma}-\sigma_{\beta}^{\eta} f_{\eta \alpha}^{\gamma}-f_{\alpha \beta}^{\eta} \sigma_{\eta}^{\gamma}\right)=0
$$

In the case the $X_{\alpha}$ commute, so that $f_{\alpha \beta}^{\gamma}=0$, this further reduces to

$$
Y_{\alpha}\left(\sigma_{\beta}^{\gamma}\right)-Y_{\beta}\left(\sigma_{\alpha}^{\gamma}\right)=0 \text {. }
$$

Note also that $\sigma$-prolongation can be defined irrespective of the involution properties of the $Y_{\alpha}$; however, reduction via $\sigma$-symmetries (see below) requires that the $Y_{\alpha}$ are an involution system ${ }^{\mathrm{k}}$, and this is not guaranteed a priori, unless the above conditions are satisfied.

A set $\left\{X_{\alpha}, \alpha=1, \ldots, r\right\}$ of vector fields in involution will be said to be a $\sigma$-symmetry module (or algebra if the $f_{\alpha \beta}^{\gamma}$ are constant) of the system $\Delta$ if their $\sigma$-prolongations $Y_{\alpha}$ satisfy

$$
Y_{\alpha}: S_{\Delta} \rightarrow \mathrm{T} S_{\Delta}
$$

As we have seen above, the possibility of using symmetries for reduction of ODEs is ultimately based on the IBDP; the same will hold true (as discussed below) for $\sigma$-symmetries, so that we need to ascertain if $\sigma$-prolonged (sets of) vector fields enjoy the IBDP. This is actually the case.

Lemma 9. A set $\left\{Y_{1}, \ldots, Y_{r}\right\}$ of $\sigma$-prolonged vector fields in involution enjoy the IBDP property. That is, if $\eta$ and $\zeta_{(k)}$ are independent common differential invariants for the $Y_{\alpha}$, so is

$$
\zeta_{(k+1)}:=\frac{D_{x} \zeta_{(k)}}{D_{x} \eta}
$$

\footnotetext{
system under commutation and obtain an involution system.

k ${ }^{\mathrm{N} o t}$ necessarily with the same involution properties; in particular, we could add new vector fields in $J^{n} M$ to close the 
G. Gaeta

Proof. We note that

$$
Y_{\alpha}\left(\zeta_{(k+1)}=\frac{\left[Y_{\alpha}\left(D_{x} \zeta_{(k)}\right] \cdot\left(D_{x} \eta\right)-\left(D_{x} \zeta_{(k)}\right) \cdot\left[Y_{\alpha}\left(D_{x} \eta\right)\right]\right.}{\left(D_{x} \eta\right)^{2}}:=\frac{\Theta}{\left(D_{x} \eta\right)^{2}}\right.
$$

so we just have to show that $\Theta=0$.

This follows by a straightforward computation (going along the lines of the one met in the proofs of Lemma 1, Lemma 3 and Lemma 5), in which we make use of the assumption that $\eta$ and $\zeta_{(k)}$ are common (scalar) differential invariants for the $Y_{\alpha}$, so that $\left.Y_{\alpha} \eta\right)=0=Y_{\alpha}\left(\zeta_{(k)}\right.$, and of (4.5). In fact, we have

$$
\begin{aligned}
\Theta & =\left[Y_{\alpha}\left(D_{x} \zeta_{(k)}\right] \cdot\left(D_{x} \eta\right)-\left(D_{x} \zeta_{(k)}\right) \cdot\left[Y_{\alpha}\left(D_{x} \eta\right)\right]\right. \\
& =\left(D_{x}\left(Y_{\alpha}\left(\zeta_{(k)}\right)\right)+\left[Y_{\alpha}, D_{x}\right]\left(\zeta_{(k)}\right)\right) \cdot\left(D_{x} \eta\right)-\left(D_{x} \zeta_{(k)}\right) \cdot\left(D_{x}\left(Y_{\alpha}(\eta)\right)+\left[Y_{\alpha}, D_{x}\right](\eta)\right) \\
& =\left(\left[Y_{\alpha}, D_{x}\right]\left(\zeta_{(k)}\right)\right) \cdot\left(D_{x} \eta\right)-\left(D_{x} \zeta_{(k)}\right) \cdot\left(\left[Y_{\alpha}, D_{x}\right](\eta)\right) \\
& =-\left(D_{x} \xi_{\alpha}+\sigma_{\alpha}{ }^{\beta} \xi_{\beta}\right) \cdot\left(D_{x} \zeta_{(k)}\right) \cdot\left(D_{x} \eta\right)+\left(D_{x} \zeta_{(k)}\right) \cdot\left(D_{x} \xi_{\alpha}+\sigma_{\alpha}^{\beta} \xi_{\beta}\right) \cdot\left(D_{x} \eta\right) \\
& =0
\end{aligned}
$$

This concludes the proof.

Remark 21. Note that if $\eta$ and $\zeta_{(k)}$ are of order $k$, then $\zeta_{(k+1)}$ is of order $k+1$, and hence independent of $\eta$ and $\zeta_{(k)}$. Thus if we have a complete basis for the set of differential invariants of order zero and one for the $Y_{\alpha}$, we can generate a basis of invariants of all orders just by differentiation. Note also that we have here assumed the $Y_{\alpha}$ are in involution; as mentioned in Remark 20, this is in general not granted.

The fact that differential invariants can be generated by means of differentiation allows to implement the same reduction strategy as for standard (or, for that matter, $\lambda$ ) symmetries. That is, we pass to symmetry-adapted variables, in which the vector fields have a trivial expression, and the differential equations system is simply independent of the new dependent variables. More precisely, in the simplest setting we have the following

Lemma 10. Let $\mathscr{X}=\left\{X_{1}, \ldots, X_{r}\right\}$ be a system (of rank $r$ ) of vector fields in involution on $M=$ $\mathbf{R} \times \mathbf{R}^{p}$; let $\mathscr{Y}=\left\{Y_{1}, \ldots, Y_{r}\right\}$ be their $\sigma$-prolongation on $J^{n} M$, still in involution and of rank $r$; and let the system $\Delta$ of $p$ ordinary differential equations of order $n>1$ admit $\mathscr{X}$ as a $\sigma$-symmetry set. Assume moreover $r=p$. Then $\Delta$ can be reduced to a system of $p$ differential equations of order $n-1$.

Proof. The $\mathscr{X}$ span a distribution of $\operatorname{rank} r=p$ in $M$, which is of dimension $p+1$; hence they admit one common invariant. By assumption, the distribution generated by $\mathscr{Y}$ has also dimension $r=p$; thus at each order $k=1, \ldots, n$ there exist exactly $p$ independent differential invariants of order $k$.

We pass to symmetry adapted coordinates $\left(y ; w^{1}, \ldots, w^{p}\right)$ such that $X_{\alpha}(y)=0$ for all $\alpha=1, \ldots, r$. Thus the geometrical invariant is simply $\eta(y, w)=y$. Call $\zeta_{a}\left(y, w, w_{(1)}\right)$ (here $\left.a=1, \ldots, p\right)$ the common differential invariants of order one, i.e. the functions $\zeta^{a}: J^{1} M \rightarrow \mathbf{R}$ satisfying $Y_{\alpha}\left(\zeta^{a}\right)=0$ for all $\alpha$ and $a$. 
Thanks to Lemma 9, the common differential invariants of order $k+1$ are obtained as

$$
\zeta_{(k)}^{a}:=D_{x}^{k} \zeta^{a} .
$$

With a new change of dependent variables, we choose these as

$$
z^{a}=\zeta^{a}\left(y ; w, w_{(1)}\right) .
$$

The system of differential equations will be written in these new variables as

$$
F^{a}\left(y ; z, \ldots, z_{(n-1)}\right)=0 ;
$$

this realizes the reduction to a system of equations of order $n-1$.

Remark 22. A similar result holds for $r<p$ (and also for equations not all of the same order); in this case, only $r$ of the $p$ differential equations are reduced to a lower order; see Theorem 4 in [17]. $\odot$

Remark 23. We also mention that the case of dynamical systems, i.e. systems of first order (autonomous) ODEs presents some special difficulties as far as $\sigma$-symmetries as concerned; these are due to the fact such a simple framework does actually introduce several degeneracies with respect to the general discussion. For a discussion of $\sigma$-symmetries (and their application) in the context of dynamical systems, see [18].

Remark 24. It should be stressed that, at difference with the standard symmetry case, obtaining solutions to the original equations from solutions to the reduced one (the so called reconstruction problem) is not trivial in this case.

In fact, we do not only have to invert the change of coordinates $(x, u) \rightarrow(y, w)$, but also have to solve the equation

$$
z^{a}(y)=\zeta^{a}\left[y ; w(y), w_{(1)}(y)\right],
$$

which now has to be considered as a system of $r$ first order differential equations for $w^{a}(y)$.

In other words, while for standard symmetries the reconstruction problem reduces to quadratures, in the present case one is faced with an auxiliary system of (in general, nonlinear and non autonomous) differential equations. Needless to say, there is no guarantee we will be able to solve this system.

In the case of $\lambda$-symmetries, we have seen that there is a simple geometric relation between $\lambda$-prolonged and standardly prolonged vector fields, see Lemma 6 . The same holds here, but as in this case $\sigma$-prolongations acts on sets of vector fields, the comparison should be made between $\sigma$ and standard prolongations of sets of vector fields.

Lemma 11. Let $\mathscr{X}=\left\{X_{1}, \ldots, X_{r}\right\}$ be a set of vector fields on $M$; and let the vector fields $\mathscr{Y}=$ $\left\{Y_{1}, \ldots, Y_{r}\right\}$ on $J^{n} M$ be their $\sigma$-prolongation. Consider also the set $\mathscr{W}=\left\{W_{1}, \ldots, W_{r}\right\}$ of vector fields on $M$ given by $W_{\alpha}=A_{\alpha}^{\beta} X_{\beta}$, with $A$ a nowhere singular matrix function on $M$; and let the vector fields $\mathscr{Z}=\left\{Z_{1}, \ldots, Z_{r}\right\}$ on $J^{n} M$ be their standard prolongation. Then, provided $A$ and $\sigma$ are related by

$$
A^{-1} D_{x} A=\sigma,
$$

we also have $Z_{\alpha}=A_{\alpha}^{\beta} Y_{\beta}$. 
G. Gaeta

Proof. It will suffice to consider first prolongations. In coordinates and with the usual shorthand notation,

$$
\begin{aligned}
X_{\alpha} & =\xi_{\alpha} \partial_{x}+\varphi_{\alpha}^{a} \partial_{a}, \\
Y_{\alpha} & =X_{\alpha}+\left(\left(D_{x} \varphi_{\alpha}^{a}-u_{x}^{a} D_{x} \xi_{\alpha}\right)+\sigma_{\alpha}^{\beta}\left(\varphi_{\beta}^{a}-u_{x}^{a} \xi_{\beta}\right)\right) \partial_{a}^{1} ; \\
W_{\alpha} & =\chi_{\alpha} \partial_{x}+\eta_{\alpha}^{a} \partial_{a}, \\
Z_{\alpha} & =W_{\alpha}+\left(D_{x} \eta_{\alpha}^{a}-u_{x}^{a} D_{x} \chi_{\alpha}\right) \partial_{a}^{1} .
\end{aligned}
$$

If now we require $W=A X$, i.e.

$$
\chi_{\alpha}=A_{\alpha}^{\beta} \xi_{\beta} ; \eta_{\alpha}^{a}=A_{\alpha}^{\beta} \varphi_{\beta}^{a},
$$

we immediately get

$$
\begin{aligned}
& D_{x} \chi_{\alpha}=\left(D_{x} A_{\alpha}^{\beta}\right) \xi_{\beta}+A_{\alpha}^{\beta}\left(D_{x} \xi_{\beta}\right), \\
& D_{x} \eta_{\alpha}^{a}=\left(D_{x} A_{\alpha}^{\beta}\right) \varphi_{\beta}^{a}+A_{\alpha}^{\beta}\left(D_{x} \varphi_{\beta}^{a}\right) .
\end{aligned}
$$

Inserting these in the expression for $Z$, we get

$$
\begin{aligned}
Z & =A_{\alpha}^{\beta}\left[\xi_{\beta} \partial_{x}+\varphi_{\beta}^{a} \partial_{a}+\left(\left(D_{x} \varphi_{\beta}^{a}-u_{x}^{a} D_{x} \xi_{\beta}\right)+\left(A^{1} D_{x} A\right)_{\alpha}^{\beta}\left(\varphi_{\beta}^{a}-u_{x}^{a} \xi_{\beta}\right)\right)\right] \partial_{a}^{1} \\
& =A_{\alpha}^{\beta} Y_{\beta}+\left[\left(D_{x} A\right)_{\alpha}^{\beta}-(A \sigma)_{\alpha}^{\beta}\right]\left(\varphi_{\beta}^{a}-u_{x}^{a} \xi_{\beta}\right) \partial_{a}^{1}
\end{aligned}
$$

Thus, provided $A$ and $\sigma$ satisfy $D_{x} A=A \sigma$, and hence (recalling $A$ is nowhere singular) satisfy (4.10), we have that $W=A X$ leads to $Y=A Z$.

Corollary. With the setting and notation of Lemma 11, denote by $\mathscr{D}$ the distribution generated by $\mathscr{Y}$, and denote by $\widehat{\mathscr{D}}$ the distribution generated by $\mathscr{Z}$. Then the distributions $\widehat{\mathscr{D}}$ and $\mathscr{D}$ coincide.

Proof. This follows immediately from Lemma 11.

Remark 25. The relations between the vector fields $X_{i}$ and $W_{i}$, and their (respectively, $\sigma$ and standard) prolongations, as given by Lemma 11, can be summarized in the form of a commutative diagram:

$$
\begin{gathered}
\left\{X_{i}\right\} \stackrel{A}{\longrightarrow}\left\{W_{i}\right\} \\
\underset{\mid}{\sigma-\text { prol }} \mid \text { prol } \\
\left\{Y_{i}\right\} \stackrel{A}{\longrightarrow}\left\{Z_{i}\right\}
\end{gathered}
$$

The relation between $A$ and $\sigma$ is given by (4.10).

Remark 26. Lemma 11 stipulates that the relation between $A$ and $\sigma$ is given by (4.10); we note that if we look at this as an equation for $A$ with a given $\sigma$, the solution is in general not unique (an explicit example is provided in [17]). Moreover - as in Remarks 10 and $16-$ unless $\sigma=D_{x} S$ for some local matrix function $S$ defined on $M, A$ can and will be a nonlocal function,

$$
A=\exp \left[\int \sigma d x\right] \text {. }
$$

Finally, we note that the sets $\left\{Y_{i}\right\}$ and $\left\{Z_{i}\right\}$ considered in Lemma 11 will in general have different involution properties. 


\subsection{Combining simple and collective twisted symmetries: $\chi$-symmetries}

We have seen that $\mu$-prolongations (and hence symmetries) are associated to matrices $\Lambda_{i}-$ or to a single matrix $\Lambda$ when we consider ODEs - acting on the vector indices $(a, b, \ldots)$ of the coefficients of each vector field; on the other hand, $\sigma$-prolongations (and hence symmetries) are associated to a matrix $\sigma$ acting on the Lie module indices $(\alpha, \beta, \ldots)$ denoting the different vector fields.

It has to be expected that the two different "twisting" of the prolongation operation can be combined with no problem, as they act on different structures. This is indeed the case, and the "combined" twisted prolongation operation (and hence symmetries) has been denoted as $\chi$-prolongation [17]. Note that $\sigma$-prolongations are (so far) only defined in the framework of a single independent variable, i.e. ODEs; hence the same applies for $\chi$-prolongations.

In order to discuss such combined twisted prolongations, we will slightly change our notation; moreover, we will limit to discuss vertical vector fields. ${ }^{1}$

We will thus consider a set of vector fields $W_{\alpha}$ on $M$, written as

$$
W_{\alpha}=\varphi_{\alpha}^{a} \partial_{a}
$$

their standard prolongations will be denoted as $Z_{\alpha}$ and will be written as

$$
Z_{\alpha}=\psi_{\alpha,(k)}^{a} \partial_{a}^{k}
$$

with $\psi_{\alpha,(0)}^{a}=\varphi_{\alpha}^{a}$ and the $\psi_{\alpha,(k)}^{a}$ obeying the standard prolongation formula (1.5).

We will then consider another set of vector fields $\widehat{X}_{\alpha}$ on $M$, written as

$$
\widehat{X}_{\alpha}=\widehat{\varphi}_{\alpha}^{a} \partial_{a}
$$

their $\mu$-prolongations will be denoted as $\widehat{Y}_{\alpha}$ and will be written as

$$
\widehat{Y}_{\alpha}=\widehat{\psi}_{\alpha,(k)}^{a} \partial_{a}^{k}
$$

with $\widehat{\psi}_{\alpha,(0)}^{a}=\widehat{\varphi}_{\alpha}^{a}$ and the $\widehat{\psi}_{\alpha,(k)}^{a}$ obeying the $\mu$-prolongation formula (3.15) (with a single independent variable, and hence a single $\Lambda$ ).

Finally we also consider a third set of vector fields $\widetilde{X}_{\alpha}$ on $M$, written as

$$
\widetilde{X}_{\alpha}=\widetilde{\varphi}_{\alpha}^{a} \partial_{a}
$$

their $\sigma$-prolongations will be denoted as $\widetilde{Y}_{\alpha}$ and will be written as

$$
\widetilde{Y}_{\alpha}=\widetilde{\psi}_{\alpha,(k)}^{a} \partial_{a}^{k}
$$

with $\widetilde{\psi}_{\alpha,(0)}^{a}=\widetilde{\varphi}_{\alpha}^{a}$ and the $\widetilde{\psi}_{\alpha,(k)}^{a}$ obeying the $\sigma$-prolongation formula (4.4).

\footnotetext{
${ }^{1}$ These can be thought as the evolutionary representative of general vector fields, see above; hence the coefficients $\varphi, \widehat{\varphi}$, $\widetilde{\varphi}$ are not only functions of the $x$ and $u$ but could depend - linearly - on the $u_{i}^{a}$ as well. 
G. Gaeta

The three sets are related by

$$
\widehat{X}_{\alpha}=A W_{\alpha} ; \widetilde{X}_{\alpha}=B_{\alpha}^{\beta} W_{\beta} .
$$

These should be meant as

$$
\widehat{\varphi}_{\alpha}^{a}=A_{b}^{a} \varphi_{\alpha}^{b}, \widetilde{\varphi}_{\alpha}^{a}=B_{\alpha}^{\beta} \varphi_{\beta}^{a} .
$$

The relations between these different sets of vector fields - which were discussed in Sections $3.2 \mathrm{e} 4.1$ - are then summarized in the following diagram

$$
\begin{aligned}
& \left\{\widehat{X}_{\alpha}\right\} \stackrel{A}{\longleftarrow}\left\{W_{\alpha}\right\} \stackrel{B}{\longrightarrow}\left\{\widetilde{X}_{\alpha}\right\} \\
& \mid \mu-\text { prol } \mid \text { prol } \\
& \left\{\widehat{Y}_{\alpha}\right\} \stackrel{A}{\longleftarrow}\left\{Z_{\alpha}\right\} \stackrel{B}{\longrightarrow}\left\{\widetilde{Y}_{\alpha}\right\}
\end{aligned}
$$

The invertible matrices $A$ and $B$ are related to the matrices $\sigma$ and $\Lambda$ (the latter identifying $\mu$ via $\mu=\Lambda \mathrm{d} x)$ by

$$
\begin{aligned}
& \Lambda=A\left(D_{x} A^{-1}\right)=-\left(D_{x} A\right) A^{-1}, \\
& \sigma=B\left(D_{x} B^{-1}\right)=-\left(D_{x} B\right) B^{-1} .
\end{aligned}
$$

The usual remarks about solution of these equations for given $\sigma$ and $\Lambda_{i}$, and the locality of the matrix functions $A$ and $B$ thus determined, would apply; see Remarks 10, 16 and 26.

We can now apply the $B$ map on the set of the $\widehat{X}_{\alpha}$, or the $A$ map on the set of the $\widetilde{X}_{\alpha}$, and obtain a new set of vector fields

$$
P_{\alpha}=A \widetilde{X}_{\alpha}=B_{\alpha}^{\beta} \widehat{X}_{\beta} .
$$

Note that the $P_{\alpha}$ will be written in coordinates as

$$
P_{\alpha}=\Phi_{\alpha}^{a} \partial_{a}=A_{b}^{a} B_{\alpha}^{\beta} \varphi_{\beta}^{b} \partial_{a} .
$$

It is obvious that the order in which the $A$ and $B$ maps are applied is inessential, as they act on different sets of indices.

We can now apply the same $A$ and $B$ on the (respectively, $\mu$ and $\sigma$ ) prolongations of the $\widehat{X}_{\alpha}$ and $\widetilde{X}_{\alpha}$, obtaining new vector fields

$$
Q_{\alpha}=A \widetilde{Y}_{\alpha}=B_{\alpha}^{\beta} \widehat{Y}_{\beta}
$$

in $J^{n} M$; these are written in coordinates as

$$
Q_{\alpha}=\Psi_{\alpha,(k)}^{a} \partial_{a}^{k}=A_{b}^{a} B_{\alpha}^{\beta} \psi_{\beta,(k)}^{b} \partial_{a}^{k},
$$

where of course $\Psi_{\alpha,(0)}^{a}=\Phi_{\alpha}^{a}$.

We will say that the $\left\{Q_{\alpha}\right\}$ are the $\chi$-prolongations of the $P_{\alpha}$. In order to keep track of the different components of this combined prolongation, we will also write $\chi=(\mu, \sigma)$. Note that $\chi$ prolongations (just like $\sigma$-prolongations) are defined on sets of vector fields, not on single ones.

We can characterize $\chi$-prolongations in terms of the recursion relations satisfied by the coefficients $\Psi_{\alpha,(k)}^{a}$ appearing in (4.19). These recursion relations are easily computed in terms of those for the $\psi_{\alpha,(k)}^{a}$ and the matrices $A$ and $B$. 
Lemma 12. The coefficients $\Psi_{\alpha,(k)}^{a}$ in the $\chi$-prolonged vector field $Q$ (4.19), with $A$ and B obeying (4.14) and (4.15), satisfy the $\chi$-prolongation formula

$$
\Psi_{\alpha,(k+1)}^{a}=D_{x} \Psi_{\alpha,(k)}^{a}+\Lambda_{b}^{a} \Psi_{\alpha,(k)}^{b}+\sigma_{\alpha}^{\beta} \Psi_{\beta,(k)}^{a} .
$$

Proof. This just follows by a simple computation. We know that for any $k \geq 0, \Psi_{\alpha,(k)}^{a}$ satisfies

$$
\Psi_{\alpha,(k)}^{a}=A_{b}^{a} B_{\alpha}^{\beta} \psi_{\beta,(k)}^{b} ;
$$

we write this, inverting the relation, as

$$
\psi_{\alpha,(k)}^{a}=\left(A^{-1}\right)_{b}^{a}\left(B^{-1}\right)_{\alpha}^{\beta} \Psi_{\beta,(k)}^{b} .
$$

In turn, we have

$$
\psi_{\alpha,(k+1)}^{a}=D_{x} \psi_{\alpha,(k)}^{a} .
$$

It follows from these, with a shorthand notation which omits to explicitly write indices, and recalling that matrices of type $A$ and $B$ commute and act on different sets of indices, that

$$
\begin{aligned}
\Psi_{(k+1)} & =A B \Psi_{(k+1)} \\
& =A B\left(D_{x} \Psi_{(k)}\right) \\
& =A B\left(D_{x}\left(A^{-1} B^{-1} \Psi_{(k)}\right)\right) \\
& =A B\left[\left(D_{x} A^{-1}\right) B^{-1} \Psi_{(k)}+A^{-1}\left(D_{x} B^{-1}\right) \Psi_{(k)}+A^{-1} B^{-1}\left(D_{x} \Psi_{(k)}\right)\right] \\
& =A B\left[\left(D_{x} A^{-1}\right) B^{-1} \Psi_{(k)}+A^{-1}\left(D_{x} B^{-1}\right) \Psi_{(k)}+A^{-1} B^{-1}\left(D_{x} \Psi_{(k)}\right)\right] \\
& =A\left(D_{x} A^{-1}\right) \Psi_{(k)}+B\left(D_{x} B^{-1}\right) \Psi_{(k)}+\left(D_{x} \Psi_{(k)}\right) .
\end{aligned}
$$

Recalling now (4.14) and (4.15), this is rewritten as

$$
\Psi_{(k+1)}=\left(D_{x} \Psi_{(k)}\right)+\Lambda \Psi_{(k)}+\sigma \Psi_{(k)} ;
$$

reintroducing indices, we have precisely (4.20).

With the introduction of $\chi$-prolongations, the diagram (4.13) is then complemented by the follow diagram:

$$
\begin{aligned}
& \left\{\widehat{X}_{\alpha}\right\} \stackrel{B}{\longrightarrow}\left\{P_{\alpha}\right\} \stackrel{A}{\longleftarrow}\left\{\widetilde{X}_{\alpha}\right\} \\
& \mid \mu-\text { prol } \mid \chi \text {-prol } \mid \sigma-\text { prol }
\end{aligned}
$$

We will as usual say that the equations $\Delta$ of order $n$ admits $P$ as a $\chi$-symmetry if its $\chi$ prolongation of order $n, Q$, satisfies $Q: S_{\Delta} \rightarrow \mathrm{T} S_{\Delta}$.

It remains to be discussed how ODEs are reduced under $\chi$-symmetries. This depends on $\chi$ prolonged vector fields enjoying the IBDP or otherwise. In fact, we know that $\Lambda$-prolonged and $\sigma$-prolonged vector fields enjoy the IBDP property, and it is easy to conclude that also $\chi$-prolonged vector fields - provided the matrix $\Lambda$ is a multiple of the identity - enjoy the IBDP property. We will actually, for the sake of completeness, prove this by explicit computation. 
G. Gaeta

Lemma 13. Let the vector fields $Q_{\alpha}$ in $J^{n} M$ be the $\chi$-prolongation of vector fields $P_{\alpha}$ in $M$, with $\chi=(\mu, \sigma)$ and $\mu=\Lambda \mathrm{d} x$. Assume moreover that $\Lambda=\lambda I$, with $\lambda: J^{1} M \rightarrow \mathbf{R}$. Then the set $\mathscr{Q}=\left\{Q_{\alpha}\right\}$ has the IBDP.

Proof. We proceed as, and with the same notation as, in the proofs of Lemmas 1, 3, 5 and 9. Using

$$
Q_{\alpha}=\Psi_{\alpha,(k)}^{a} \partial_{a}^{k} ; \quad D_{x}=\partial_{x}+u_{(k+1)}^{a} \partial_{a}^{k},
$$

we immediately get

$$
\left[Q_{\alpha}, D_{x}\right]=\left(\Psi_{\alpha,(k+1)}^{a}-\left(D_{x} \Psi_{\alpha,(k)}^{a}\right)\right) \partial_{a}^{k}
$$

With (4.20), this yields

$$
\left[Q_{\alpha}, D_{x}\right]=\left(\Lambda_{b}^{a} \Psi_{\alpha,(k)}^{b}+\sigma_{\alpha}^{\beta} \Psi_{\beta,(k)}^{a}\right) \partial_{a}^{k} .
$$

Using now the hypothesis $\Lambda=\lambda I$, this gives simply

$$
\left[Q_{\alpha}, D_{x}\right]=\left(\lambda \Psi_{\alpha,(k)}^{a}+\sigma_{\alpha}^{\beta} \Psi_{\beta,(k)}^{a}\right) \partial_{a}^{k}=\lambda Q_{\alpha}+\sigma_{\alpha}^{\beta} Q_{\beta} .
$$

We will introduce the matrix

$$
\rho:=\lambda I+\sigma
$$

the previous relation is thus written as

$$
\left[Q_{\alpha}, D_{x}\right]=\rho_{\alpha}^{\beta} Q_{\beta} .
$$

To show the IBDP, we have to show that for any common differential invariants $\eta$ and $\zeta_{(k)}$, and for an $\alpha$, it results $Q_{\alpha}\left[\left(D_{x} \zeta_{(k)}\right) /\left(D_{x} \eta\right)\right]=0$, i.e. that

$$
\Theta_{\alpha}:=\left(Q_{\alpha}\left(D_{x} \zeta_{(k)}\right)\right) \cdot\left(D_{x} \eta\right)-\left(D_{x} \zeta_{(k)}\right) \cdot\left[Q_{\alpha}\left(D_{x} \eta\right)\right]=0 .
$$

This can be rewritten as

$$
\begin{aligned}
\Theta_{\alpha} & =\left(Q_{\alpha}\left(D_{x} \zeta_{(k)}\right)\right) \cdot\left(D_{x} \eta\right)-\left(D_{x} \zeta_{(k)}\right) \cdot\left[Q_{\alpha}\left(D_{x} \eta\right)\right] \\
& =\left(\left[Q_{\alpha}, D_{x}\right]\left(\zeta_{(k)}\right)-D_{x}\left(Q_{\alpha}\left(\zeta_{(k)}\right)\right)\right) \cdot\left(D_{x} \eta\right)-\left(D_{x} \zeta_{(k)}\right) \cdot\left(\left[Q_{\alpha}, D_{x}\right](\eta)-D_{x}\left(Q_{\alpha}(\eta)\right)\right) \\
& =\left(\left[Q_{\alpha}, D_{x}\right]\left(\zeta_{(k)}\right)\right) \cdot\left(D_{x} \eta\right)-\left(D_{x} \zeta_{(k)}\right) \cdot\left(\left[Q_{\alpha}, D_{x}\right](\eta)\right) \\
& =\rho_{\alpha}^{\beta}\left[\left(Q_{\beta}\left(\zeta_{(k)}\right)\right) \cdot\left(D_{x} \eta\right)-\left(D_{x} \zeta_{(k)}\right) \cdot\left(Q_{\beta}(\eta)\right)\right] .
\end{aligned}
$$

We have used the hypothesis $Q_{\alpha}\left(\zeta_{(k)}\right)=0=Q_{\alpha}(\eta)$ for all $\alpha$. Using this once again, we conclude that $\Theta_{\alpha}=0$ for all $\alpha$; and hence that the IBDP holds for $\chi$-prolongations, under the condition $\Lambda=\lambda I$.

\section{The geometric meaning of twisted symmetries}

We have seen that there are different types of twisted symmetries; some of these enjoy the IBDP, and can then be used to perform symmetry reduction of PDEs.

It is natural to wonder what is the characteristic singling out the deformations of the prolongation operation which still allow to perform reduction. The answer is actually simpler when we look at 
combined twisted symmetries, as in the case of simple twisted prolongations the situation is in some way a degenerate one and some features are less apparent.

The condition for a vector field $X$ to be a symmetry (in its different declinations) of a system $\Delta$ is that its prolongation (standard or twisted) is tangent to the solution manifold $S_{\Delta}$; in the same way, the condition for a system of vector fields $\mathscr{X}=\left\{X_{\alpha}\right\}$ to be a module (possibly an algebra) of symmetries is that their prolongations (standard or twisted) $\mathscr{Y}=\left\{Y_{\alpha}\right\}$ are tangent to $S_{\Delta}$.

Note that the tangency condition is irrespective of a global nonzero factor (possibly depending on the point in $J^{n} M$, i.e. a smooth function $f: J^{n} M \rightarrow \mathbf{R}$ ). That is, if $Y$ is tangent to $S_{\Delta}$, so is $f \cdot Y$; and similarly for systems of vector fields.

We can reverse the point of view, and express the same tangency condition putting the accent on $Y$ rather than on $S_{\Delta}$.

Definition. A vector field $X$ (a system $\mathscr{X}=\left\{X_{\alpha}\right\}$ of vector fields) is a symmetry for $\Delta$ if $S_{\Delta}$ is an integral manifold for the distribution generated by the prolongation $Y$ (the prolongations $\mathscr{Y}=\left\{Y_{\alpha}\right\}$ ) of $X$ (of the $X_{\alpha}$ ).

It is obvious that this Definition is completely equivalent to the standard one. However, focusing on distributions shows immediately that we can change generators - i.e. the vector field $Y$ or the vector fields $Y_{\alpha}$ - provided the distribution remains the same.

In the case of a single generator $Y$, the only way to change generator keeping the same distribution is by multiplying $Y$ by a nowhere zero ${ }^{\mathrm{m}}$ factor $A\left(x, u, \ldots, u_{(n)}\right)$. If we want moreover that the restriction of $Y$ to any one of the jet spaces $J^{k} M$ (for $k=0, \ldots, n$ ) is well defined, i.e. that $Y$ is projectable in $J^{k} M$ (in this case we say just it is projectable for short), then we have to require that $A$ does not depend on the derivatives of $u$, i.e. that $A=A(x, u)$.

If we look at vector fields $Y$ in $J^{n} M$ which are obtained in this way from a vector field $Z$ which is a standardly prolonged vector field (say the standard prolongation of $W$, a vector field in $M$ ), i.e. $Y=A Z$, then $Y$ is the $\Lambda$-prolongation of a vector field in $M$. More precisely, we have the

Lemma 14. Let $W$ be a vector field in $M$, and $Z$ its standard prolongation to $J^{n} M$. Let $Y=\gamma Z$, with $\gamma(x, u)$ a nowhere zero function. Then $Y$ is the $\Lambda$-prolongation, with $\Lambda=\lambda I$, of a vector field $X$ in $M$, given by $X=\gamma^{-1} W$; the functions $\gamma$ and $\lambda$ are related by

$$
\lambda=\gamma\left(D_{x} \gamma^{-1}\right)=-\left(D_{x} \gamma\right) \gamma^{-1}
$$

Proof. The proof actually mostly amounts to repeating (in reverse) a computation already performed; see Lemma 4 and Remark 8, but we give it for the sake of completeness.

Note that $Z$ is surely projectable, being the standard prolongation of a Lie-point vector field; it follows immediately that $Y$ is also projectable. We write it in coordinates, with the usual notation, as

$$
Y=\widehat{\xi} \partial_{x}+\widehat{\psi}_{(k)}^{a} \partial_{a}^{k}
$$

Here $\widehat{\xi}=\widehat{\xi}(x, u)$, while $\widehat{\psi}_{(k)}^{a}=\widehat{\psi}_{(k)}^{a}\left(x, u, \ldots, u_{(k)}\right)$, and we also write $\widehat{\psi}_{(0)}^{a}=\widehat{\varphi}^{a}$.

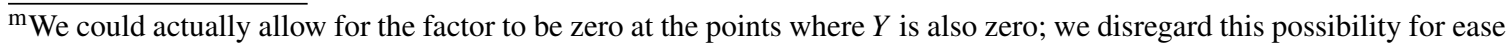
of writing, but the essential point (also below) is that the rank of the distribution should remain the same at each point. 
G. Gaeta

By assumption, $Z$ can be written as

$$
Z=\xi \partial_{x}+\psi_{(k)}^{a} \partial_{a}^{k},
$$

where the $\psi_{(k)}^{a}$ satisfy the prolongation formula (1.5), with $\psi_{(0)}^{a}=\varphi^{a}$; this is the prolongation of

$$
W=\xi \partial_{x}+\varphi^{a} \partial_{a}
$$

We also know that $Y=\gamma Z$, i.e.

$$
\widehat{\xi}=\gamma \xi, \quad \widehat{\psi}_{(k)}^{a}=\gamma \psi_{(k)}^{a}
$$

Therefore, using (1.5), we get

$$
\begin{aligned}
\widehat{\psi}_{(k+1)}^{a} & =\gamma \psi_{(k+1)}^{a}=\gamma\left[\left(D_{x} \psi_{(k)}^{a}\right)-u_{(k+1)}^{a}\left(D_{x} \xi\right)\right] \\
& =\gamma\left[\left(D_{x}\left(\gamma^{-1} \widehat{\psi}_{(k)}^{a}\right)\right)-u_{(k+1)}^{a}\left(D_{x}\left(\gamma^{-1} \widehat{\xi}\right)\right)\right] \\
& =\left[\left(D_{x} \widehat{\psi}_{(k)}^{a}\right)-u_{(k+1)}^{a}\left(D_{x} \widehat{\xi}\right)\right]+\gamma\left(D_{x} \gamma^{-1}\right)\left[\widehat{\psi}_{(k)}^{a}-u_{(k+1)}^{a} \widehat{\xi}\right] .
\end{aligned}
$$

This means that the $\widehat{\psi}_{(k)}^{a}$ obey the $\Lambda$-prolongation formula (3.3) with

$$
\Lambda=\left[\gamma\left(D_{x} \gamma^{-1}\right)\right] I=-\left[\left(D_{x} \gamma\right) \gamma^{-1}\right] I
$$

as stated in the Lemma.

Remark 27. The fact that $\lambda$-prolongations are the only vector fields ${ }^{\mathrm{n}}$ which are collinear to (and hence admit the same invariants as) a standardly prolonged vector field was first noticed by Pucci and Saccomandi [60] in a seminal paper which set the basis for a geometrical understanding of $\lambda$-symmetries and then of other types of twisted symmetries.

The following result is very simple but expresses the relations between standard and $\lambda$ symmetries.

Lemma 15. Let $X, Y, W, Z$ be as in Lemma 14 above. Then $X$ is a $\Lambda$-symmetry of the differential equations $\Delta$, with $\Lambda=\lambda I$, if and only if $W$ is a standard symmetry of $\Delta$.

Proof. The vector fields $Y$ and $Z$ span the same distribution, hence the result follows from the Definition above.

The results obtained above for simple twisted symmetries as $\Lambda$-symmetries immediately generalizes (basically with the same proof) to the case of collective twisted symmetries as $\sigma$-symmetries.

Lemma 16. Let $\mathscr{W}=\left\{W_{1}, \ldots, W_{r}\right\}$ be a set of vector fields in involution in $M$, and $\mathscr{Z}=\left\{Z_{1}, \ldots, Z_{r}\right\}$ the set of their standard prolongations to $J^{n} M$. Let $Y_{\alpha}=\Gamma_{\alpha}^{\beta} Z_{\beta}$, with $\Gamma=\Gamma(x, u)$ a nowhere singular matrix function on $M$. Then $\mathscr{Y}$ is the $\sigma$-prolongation of a set of vector fields $\mathscr{X}=\left\{X_{1}, \ldots, X_{r}\right\}$ in $M$, given by $X_{\alpha}=\left(\Gamma^{-1}\right)_{\alpha}^{\beta} W_{\beta}$; the matrix functions $G$ and $\sigma$ are related by

$$
\sigma=\Gamma\left(D_{x} \Gamma^{-1}\right)=-\left(D_{x} \Gamma\right) \Gamma^{-1} .
$$

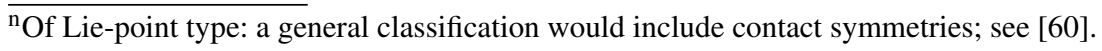


Proof. We proceed as in the proof to Lemma 14; again the proof actually mostly amounts to repeating (in reverse) a computation already performed, see Lemma 11.

The $Z_{\alpha}$ are surely projectable, and so are the $Y_{\alpha}$. We write them, with the usual notation, as

$$
Y_{\alpha}=\widetilde{\xi}_{\alpha} \partial_{x}+\widetilde{\psi}_{\alpha,(k)}^{a} \partial_{a}^{k}
$$

We also write $\widetilde{\psi}_{\alpha,(0)}^{a}=\widetilde{\varphi}_{\alpha}^{a}$.

By assumption, $Z_{\alpha}$ can be written as

$$
Z_{\alpha}=\xi_{\alpha} \partial_{x}+\psi_{\alpha,(k)}^{a} \partial_{a}^{k}
$$

with coefficients $\psi_{\alpha,(k)}^{a}$ satisfying the prolongation formula (1.5), with $\psi_{\alpha,(0)}^{a}=\varphi_{\alpha}^{a}$; this is the prolongation of

$$
W=\xi_{\alpha} \partial_{x}+\varphi_{\alpha}^{a} \partial_{a}
$$

We also know that $Y=\Gamma Z$, i.e.

$$
\widetilde{\xi}_{\alpha}=\Gamma_{\alpha}^{\beta} \xi_{\beta}, \quad \widetilde{\psi}_{\alpha,(k)}^{a}=\Gamma_{\alpha}^{\beta} \psi_{\beta,(k)}^{a} .
$$

Therefore, using again (1.5), we get

$$
\begin{aligned}
\widetilde{\psi}_{\alpha,(k+1)}^{a} & =\Gamma_{\alpha}^{\beta} \psi_{\alpha,(k+1)}^{a} \\
& =\Gamma_{\alpha}^{\beta}\left[\left(D_{x} \psi_{\beta,(k)}^{a}\right)-u_{(k+1)}^{a}\left(D_{x} \xi_{\beta}\right)\right] \\
& =\Gamma_{\alpha}^{\eta}\left[\left(D_{x}\left(\left(\Gamma^{-1}\right)_{\eta}^{\beta} \widetilde{\psi}_{\beta,(k)}^{a}\right)\right)-u_{(k+1)}^{a}\left(D_{x}\left(\left(\Gamma^{-1}\right)_{\eta}^{\beta} \widetilde{\xi}_{\beta}\right)\right)\right] \\
& =\left[\left(D_{x} \widetilde{\psi}_{\alpha,(k)}^{a}\right)-u_{(k+1)}^{a}\left(D_{x} \widetilde{\xi}_{\alpha}\right)\right]+\Gamma_{\alpha}^{\eta}\left(D_{x}\left(\Gamma^{-1}\right)_{\eta}^{\beta}\right)\left[\widetilde{\psi}_{\beta,(k)}^{a}-u_{(k+1)}^{a} \widetilde{\xi}_{\beta}\right] .
\end{aligned}
$$

This means that the $\widetilde{\psi}_{\alpha,(k)}^{a}$ obey the $\sigma$-prolongation formula (4.4) with $\sigma$ as in (5.3).

Lemma 17. Let $\mathscr{X}, \mathscr{Y}, \mathscr{W}, \mathscr{Z}$ be as in Lemma 16 above. Then $\mathscr{X}$ is a set of $\sigma$-symmetries of the differential equations $\Delta$ if and only if $\mathscr{W}$ is a set of standard symmetries of $\Delta$.

Proof. The set of vector fields $\left\{Y_{\alpha}\right\}$ and $\left\{Z_{\alpha}\right\}$ span the same distribution, hence the result follows from the Definition above.

Remark 28. One could also obtain similar results for $\chi$-symmetries; however we have seen above that these are of interest for symmetry reduction of ODEs only in the case where $\Lambda=\lambda I$; in this case $\chi$-prolongations with $\chi=(\Lambda, \widehat{\sigma})$ are de facto equivalent to sigma-prolongations with $\sigma=(\lambda I+\widehat{\sigma})$, as seen also in the proof to Lemma 13.

Finally, we will conclude our discussion with some words concerning the relation of these results with the gauge-theoretic properties of twisted symmetries.

The fact that twisted symmetries are (locally) gauge-equivalent to standard ones, or more precisely (and more generally) that vector fields obtained via twisted prolongations are gauge equivalent to vector fields obtained via standard prolongations, can now be readily understood.

In fact, our Definition 1 focuses on distributions rather on (their generating) vector fields; the gauge transformations which appeared in discussing several types of twisted prolongations are transformations which act on the generating sets, i.e. on vector fields, changing them but keeping the distribution they generate invariant. In this sense, the appearance of such gauge-theoretic 
properties is entirely natural from the point of view of distributions - albeit it might seem rather mysterious when we focus on single vector fields.

It should be stressed that our results concerning gauge equivalence of twisted and standard symmetries were obtained under the assumption that the matrices embodying the gauge transformations are invertible at all points; if this assumption fails, the equivalence is only local, in open neighborhoods not including singular points. The singular or nonsingular nature of the concerned matrices is not left to our choice, but is commanded by the function $\lambda$, or the matrix $\sigma$, or the matrices $\Lambda_{i}$.

Actually, it is precisely the case where the gauge equivalence with standard symmetries is only local to be the most interesting, in the sense in this case the consideration of twisted symmetries allows to obtain really new results. ${ }^{\circ}$

\section{Other topics}

We should finally mention some other relevant topics, also investigated in relation to twisted symmetries, which have not been discussed in the present paper, and for which we refer to the literature.

First of all, twisted symmetries can also be formulated as a generalization of orbital symmetries $[29,65,66]$; this is discussed in detail in [19], see also [12, 17, 18, 20].

In a very recent note [38], Morando investigated the relation with reducible structures, extending the approach mentioned in Remark 3 above and exploring the relation with Frobenius reduction.

The relation between twisted symmetries (in particular, $\lambda$-symmetries), first integrals and integrating factors $[4,5,12,13,28,36,48,49]$, as well as that between twisted symmetries (in particular, $\lambda$-symmetries) and Jacobi Last Multiplier $[51,53]$ has been studied by several authors .

Finally, we mention that twisted symmetries (in particular, once again, $\lambda$-symmetries) and their applications have also been studied for discrete equations [33,34].

\section{Conclusions}

We have reviewed recent advances in twisted prolongations of vector fields and twisted symmetries of (systems of) differential equations, complementing our previous review paper [23] and providing an up-to-date discussion.

Recent advances concerned the introduction, beside simple ones, of collective twisted prolongations. These in turn led to focus on distributions rather than on single vector fields; this focus on distributions - even in the case of those generated by a single vector fields, as is the case for simple twisted symmetries - helped in turn in getting an understanding of the geometry of (collective, but also simple) twisted prolongations, and explained why we have local gauge equivalence between standard and twisted symmetries.

\section{References}

[1] D.V. Alekseevsky, A.M. Vinogradov and V.V. Lychagin, Basic ideas and concepts of differential geometry, Springer 1991

[2] A. Barco and G.E. Prince, "Solvable symmetry structures in differential form", Acta Appl. Math. 66 (2001), 89-121

${ }^{\mathrm{o}}$ It should also be recalled that, as discussed in Sect.3.3, the gauge equivalence can also connect twisted symmetries expressed by Lie-point vector fields to standard symmetries (in the sense of symmetries arising from standard prolongation) which are expressed by generalized, rather than Lie-point, vector fields. 
[3] P. Basarab-Horwath, "Integrability by quadratures for systems of involutive vector fields", Ukr. Math. J. 43 (1992), 1236-1242

[4] A. Bhuvaneswari, R.A. Kraenkel and M. Senthilvelan, "Application of the $\lambda$-symmetries approach and time independent integral of the modified Emden equation", Nonlin. Anal. RWA 13 (2012), 1102-1114

[5] A. Bhuvaneswari, R.A. Kraenkel and M. Senthilvelan, "Lie-point symmetries and the time-independent integral of the damped harmonic oscillator", Physica Scripta 83 (2011), 055005

[6] J.F. Carinena, M. Del Olmo and P. Winternitz, "On the relation between weak and strong invariance of differential equations", Lett. Math. Phys. 29 (1993), 151-163

[7] D. Catalano Ferraioli, "Nonlocal aspects of $\lambda$-symmetries and ODEs reduction", J. Phys. A: Math. Theor. 40 (2007), 5479-5489

[8] D. Catalano Ferraioli and P. Morando, "Local and nonlocal solvable structures in the reduction of ODEs", J. Phys. A: Math. Theor. 42 (2009), 035210 (15pp)

[9] D. Catalano Ferraioli and P. Morando, "Exploiting solvable structures in the integration of variational ordinary differential equations", preprint 2014

[10] G. Cicogna, "Reduction of systems of first-order differential equations via $\Lambda$-symmetries", Phys. Lett. A 372 (2008), 3672-3677

[11] G. Cicogna, "Symmetries of Hamiltonian equations and $\Lambda$-constants of motion", J. Nonlin. Math. Phys. 16 (2009), 43-60

[12] G. Cicogna, "Generalized notions of symmetry of ODEs and reduction procedures", Mathematical Methods in the Applied Sciences (2013), DOI:10.1002/mma.2937

[13] G. Cicogna, "On the connections between symmetries and conservation rules of dynamical systems", Math. Meth. Appl. Sci. 36 (2013), 208-215

[14] G. Cicogna and G. Gaeta, "Partial Lie-point symmetries of differential equations", J. Phys. A 34 (2001), 491-512

[15] G. Cicogna and G. Gaeta, Symmetry and perturbation theory in nonlinear dynamics, Springer 1999

[16] G. Cicogna, G. Gaeta and P. Morando, "On the relation between standard and $\mu$-symmetries for PDEs", J. Phys. A 37 (2004), 9467-9486

[17] G. Cicogna, G. Gaeta and S. Walcher, "A generalization of $\lambda$-symmetry reduction for systems of ODEs: $\sigma$-symmetries”, J. Phys. A: Math. Theor. 45 (2012) 355205 (29 pp)

[18] G. Cicogna, G. Gaeta and S. Walcher: "Dynamical systems and $\sigma$-symmetries", J. Phys. A 46 (2013), 235204 (23pp)

[19] G. Cicogna, G. Gaeta and S. Walcher, "Orbital reducibility and a generalization of lambda symmetries", J. Lie Theory 23 (2013), 357-381

[20] G. Cicogna, G. Gaeta and S. Walcher, "Side conditions for ordinary differential equations", preprint 2014, to appear in J. Lie Theory

[21] P.A. Clarkson and M.D. Kruskal, "New similarity solutions of teh Boussinesq equation”, J. Math. Phys. 30 (1989), 2201-2213

[22] G. Gaeta, Nonlinear symmetries and nonlinear equations, Kluwer, Dordrecht 1994

[23] G. Gaeta, "Twisted symmetries of differential equations", J. Nonlin. Math. Phys., 16-S (2009), 107-136

[24] G. Gaeta, "Gauge fixing and twisted prolongations", J. Phys. A 44 (2011), 325203 (9pp)

[25] G. Gaeta and P. Morando, "On the geometry of lambda-symmetries and PDE reduction", J. Phys. A 37 (2004), 6955-6975

[26] C. Godbillon, Géométrie Différentielle et Mécanique Analitique, Hermann 1969

[27] K.S. Govinder and P.G.L. Leach, "On the determination of non-local symmetries" J. Phys. A: Math. Gen. 28 (1995), 5349-5359

[28] G. Gun and T. Ozer, "First Integrals, integrating factors, and invariant solutions of the path equation based on Noether and $\lambda$-symmetries", Abstr. Appl. Anal. 2013 (2013), 284653

[29] K.P. Hadeler and S. Walcher, "Reducible ordinary differential equations", J. Nonlinear Sci. 16 (2006), 583-613

[30] T. Hartl and C. Athorne, "Solvable structures and hidden symmetries", J. Phys. A: Math. Gen. 27 (1994), 3463-3471

[31] R. Hermann, Differential Geometry and the Calculus of Variations, Academic Press (New York) 1968 
G. Gaeta

[32] I.S. Krasil'schik and A.M. Vinogradov, Symmetries and conservation laws for differential equations of mathematical physics, A.M.S. 1999

[33] D. Levi, M.C. Nucci and M.A. Rodriguez, " $\lambda$-symmetries for the reduction of continuous and discrete equations", Acta Appl. Math 122 (2012), 311-321

[34] D. Levi and M.A. Rodriguez, " $\lambda$-symmetries for discrete equations", arXiv:1004.4808

[35] D. Levi and P. Winternitz, "Non-classical symmetry reduction: example of the Boussinesq equation", J. Phys. A 22 (1989), 2915-2924

[36] R. Mohanasubha et al., "Interplay of symmetries, null forms, Darboux polynomials, integrating factors and Jacobi multipliers in integrable second-order differential equations", Proc. Royal Soc. A 470 (2014), 20130656

[37] P. Morando, "Deformation of Lie derivative and $\mu$-symmetries", J. Phys. A 40 (2007), 11547-11560

[38] P. Morando, "Reduction by $\lambda$-symmetries and $\sigma$-symmetries: a Frobenius approach", preprint 2014

[39] P. Morando and S. Sammarco, "Variational problems with symmetries: a Pfaffian system approach", Acta Appl. Math. 120 (2012), 255-274

[40] C. Muriel and J.L. Romero, "New methods of reduction for ordinary differential equations", IMA J. Appl. Math. 66 (2001), 111-125

[41] C. Muriel and J.L. Romero, " $C^{\infty}$ symmetries and nonsolvable symmetry algebras”, IMA J. Appl. Math. 66 (2001), 477-498

[42] C. Muriel and J.L. Romero, "Prolongations of vector fields and the invariants-by-derivation property", Theor. Math. Phys. 113 (2002), 1565-1575

[43] C. Muriel and J.L. Romero, " $C^{\infty}$-symmetries and integrability of ordinary differential equations"; $p$. 143-150 in Proceedings of the I Colloquium on Lie theory and applications (Vigo), 2002

[44] C. Muriel and J.L. Romero, " $C^{\infty}$ symmetries and reduction of equations without Lie point symmetries", J. Lie Theory 13 (2003) 167-188

[45] C. Muriel and J.L. Romero, " $C^{\infty}$-symmetries and nonlocal symmetries of exponential type, IMA J. Appl. Math. 72 (2007) 191-205

[46] C. Muriel and J.L. Romero, "Integrating factors and lambda-symmetries", J. Nonlin. Math. Phys. 15S3 (2008), 300-309

[47] C. Muriel and J.L. Romero, "First integrals, integrating factors and $\lambda$-symmetries of second-order differential equations", J. Phys. A 42 (2009), 365207

[48] C. Muriel and J.L. Romero, "A $\lambda$-symmetry-based method for the linearization and determination of first integrals of a family of second-order ordinary differential equations", J. Phys. A 44 (2011), 245201

[49] C. Muriel and J.L. Romero, "Second-order differential equations with first integrals of the form $C(t)+$ $1 /(A(t, x) x+B(t, x)), J$. Nonlin. Math. Phys. 18-S1 (2011), 237-250

[50] C. Muriel and J.L. Romero, "Nonlocal symmetries, telescopic vector fields and $\lambda$-symmetries of ordinary differential equations", Symmetry, Integrability and Geometry: Methods and Applications 8 (2012), 106

[51] C. Muriel and J.L. Romero, "The $\lambda$-symmetry reduction method and Jacobi last multipliers", Comm. Nonlin. Science Num. Sim. 19 (2014), 807-820

[52] C. Muriel, J.L. Romero and P.J. Olver, "Variational $C^{\infty}$ symmetries and Euler-Lagrange equations", $J$. Diff. Eqs. 222 (2006), 164-184

[53] M.C. Nucci and D. Levi, “ $\lambda$-symmetries and Jacobi last multiplier”, Nonlin. Anal. RWA 14 (2013), 1092-1101

[54] F. Oliveri, "Lie symmetries of differential equations: classical results and recent contributions", Symmetry 2 (2010), 658-706

[55] P.J. Olver, Application of Lie groups to differential equations, Springer 1986

[56] P.J. Olver, Equivalence, Invariants and Symmetry, Cambridge University Press 1995

[57] P.J. Olver and $\mathrm{Ph}$. Rosenau, "The construction of special solutions to partial differential equations", Phys. Lett. A 114 (1986), 107-112

[58] P.J. Olver and Ph. Rosenau, "Group-invariant solutions of differential equations", SIAM J. Appl. Math. 47 (1987), 263-278 
[59] E. Pucci and G. Saccomandi, "On the weak symmetry groups of partial differential equations", J. Math. Anal. Appl. 163 (1992), 588-598

[60] E. Pucci and G. Saccomandi, "On the reduction methods for ordinary differential equations", J. Phys. A 35 (2002), 6145-6155

[61] R.W. Sharpe, Differential Geometry, Springer 1997

[62] J. Sherring and G. Prince, "Geometric aspects of reduction of order", Trans. Am. Math. Soc. 334 (1992), 433-453

[63] H. Stephani, Differential equations. Their solution using symmetries, Cambridge University Press 1989

[64] S. Sternberg Lectures on Differential Geometry, Chelsea 1983

[65] S. Walcher, "Multi-parameter symmetries of first order ordinary differential equations", J. Lie Theory 9 (1999), 249-269

[66] S. Walcher, "Orbital symmetries of first order ODEs", in Symmetry and Perturbation Theory (SPT98), Editors: Degasperis A and Gaeta G, World Scientific, Singapore (1999): 96-113 